NBER WORKING PAPER SERIES

\title{
WHO IS SCREENED OUT? APPLICATION COSTS AND THE TARGETING OF DISABILITY PROGRAMS
}

\author{
Manasi Deshpande \\ Yue Li \\ Working Paper 23472 \\ http://www.nber.org/papers/w23472 \\ NATIONAL BUREAU OF ECONOMIC RESEARCH \\ 1050 Massachusetts Avenue \\ Cambridge, MA 02138 \\ June 2017, Revised September 2017
}

We thank many offices and individuals at the Social Security Administration for providing access to data; Sirisha Anne, Thuy Ho, Bill Lancaster, Linda Martin, and especially Françoise Becker of SSA for data work; and many current and former Social Security Administration employees for sharing their institutional knowledge. We thank Christopher Cronin, Michael Dinerstein, Amy Finkelstein, John Friedman, Michael Greenstone, Magne Mogstad, Casey Mulligan, Derek Neal, Hoai-Luu Nguyen, Jesse Shapiro, Robert Topel, and participants at several seminars for helpful comments, and Michele Carter and Nina Nguyen for excellent research assistance. This research was supported by the U.S. Social Security Administration through grant \#1 DRC12000002-05 to the National Bureau of Economic Research as part of the SSA Disability Research Consortium. The findings and conclusions expressed are solely those of the authors and do not represent the views of SSA, any agency of the Federal Government, or the NBER. Each author declares that she has no relevant or material financial interests that relate to the research described in this paper. The views expressed herein are those of the authors and do not necessarily reflect the views of the National Bureau of Economic Research.

NBER working papers are circulated for discussion and comment purposes. They have not been peer-reviewed or been subject to the review by the NBER Board of Directors that accompanies official NBER publications.

(C) 2017 by Manasi Deshpande and Yue Li. All rights reserved. Short sections of text, not to exceed two paragraphs, may be quoted without explicit permission provided that full credit, including $\odot$ notice, is given to the source. 
Who Is Screened Out? Application Costs and the Targeting of Disability Programs

Manasi Deshpande and Yue Li

NBER Working Paper No. 23472

June 2017, Revised September 2017

JEL No. H2,I3

\begin{abstract}
$\underline{\text { ABSTRACT }}$
The application process is critical to the targeting of disability programs because disability, relative to other tags, is difficult to observe and costly to verify. We study the effect of application costs on the targeting of disability programs using the closings of Social Security Administration field offices, which provide assistance with filing disability applications. Using administrative data from the Social Security Administration, we find that field office closings lead to large and persistent reductions in the number of disability recipients and reduce targeting efficiency based on current eligibility standards. The number of disability recipients declines by $13 \%$ in surrounding areas, with the largest effects for applicants with moderately severe conditions, low education levels, and low pre-application earnings. Evidence on channels suggests that most of the reduction in applications is attributable to increased congestion at neighboring offices rather than increased travel times or costs of information gathering.
\end{abstract}

Manasi Deshpande

Department of Economics

University of Chicago

1126 E 59th St.

Chicago, IL 60637

and NBER

mdeshpande@uchicago.edu

Yue Li

Department of Economics

SUNY Albany

1400 Washington Avenue

Albany, NY 12222

USA

yli49@albany.edu

An online appendix is available at http://www.nber.org/data-appendix/w23472 
Disability programs are large and expanding rapidly across the developed world. Social Security Disability Insurance (SSDI or DI), the insurance program for disabled workers in the United States, provided cash benefits and Medicare to nearly 9 million workers in 2015, up from 5 million in 2000. In 2015, Supplemental Security Income (SSI) provided cash welfare and Medicaid eligibility to nearly 7 million low-income, disabled Americans, including 1.4 million children. ${ }^{1}$ These programs are intended to provide disability benefits to those - and only those -individuals who have severe disabilities and are in need of assistance. The primary system for targeting disability programs is the disability determination process, in which adjudicators determine whether an individual meets the medical eligibility criteria for these programs. However, even before potential applicants encounter the disability determination system, the cost of applying for disability programs may affect whether they decide to apply and, as a result, whether they receive disability benefits. To apply for disability, individuals must consider whether they are eligible, submit extensive paperwork, and provide access to medical records. The effect of these application costs on the targeting of disability programs is ambiguous: hassles could screen out either those most in need or least in need, depending on how potential applicants respond to these costs.

The application process is especially important for the targeting of disability programs because disability is difficult to observe and costly to verify. For programs based only on income or earnings, the government can use earnings and income data from the tax system or other sources to automatically enroll households or notify them of their eligibility. In contrast, the government does not collect data on health or disability status outside of the disability determination process. If individuals with severe disabilities do not apply because the application process is too costly, the government has few other ways to identify them and provide benefits. Conversely, the government may want to design an application process that discourages low-severity individuals from applying, since recent evidence indicates that the application process leads to earnings decay for applicants who are ultimately rejected (Autor, Maestas, Mullen and Strand (2015)).

In this paper, we address how application costs affect the targeting of disability programs. Nichols and Zeckhauser (1982) argue that application costs can improve targeting by screening out high-ability individuals with a high opportunity cost of time. In their model, the loss in productive efficiency from application hassles is more than offset by the gain in targeting efficiency. How-

\footnotetext{
${ }^{1}$ Annual Statistical Report on the Social Security Disability Insurance Program, 2015; SSI Annual Statistical Report, 2015. SSI provides categorical Medicaid eligibility in most states, except for ten states that use stricter criteria to determine Medicaid eligibility for the disabled; seven other states require SSI recipients to submit a separate Medicaid application to the state. SSI also provides benefits to low-income elderly individuals.
} 
ever, evidence from behavioral economics suggests that hassles may discourage those most in need (Bertrand, Mullainathan and Shafir (2004)). We provide the first empirical test of this debate in the context of disability programs using variation in the timing of closings of Social Security Administration (SSA) field offices, which provide assistance with filing disability applications. Using detailed administrative data on disability applications and applicant characteristics, we estimate the effect of an increase in application costs induced by field office closings on the number and composition of disability applicants and recipients. We employ a difference-in-differences strategy that compares the number and composition of disability applicants and recipients in areas that experience the closing of their nearest field office to areas that do not experience a closing until several years later, before and after the closing. Using detailed SSA data on field office characteristics, we also evaluate the relative importance of different types of application costs induced by the closings, including travel time to assistance and congestion at neighboring field offices.

We find that field office closings, in addition to reducing take-up substantially, reduce the targeting efficiency of disability programs based on current eligibility standards. The closings reduce the number of disability applications by 11 percent (12 applications per ZIP per quarter) and the number of recipients by 13 percent (6 "allowances," or final approvals, per ZIP per quarter) in surrounding areas, meaning that they disproportionately discourage applications from individuals who would have been allowed onto the program if they had applied. The closings have the largest discouragement effects for those with moderately severe conditions, low education levels, and low pre-application earnings. The discouragement effects persist for at least two years after the closing, and they also occur in areas surrounding neighboring offices since those offices become more congested after the closing. The magnitude of the effects is large, suggesting an implied value of time of $\$ 100$ per hour for disability applicants.

To better understand these effects, we examine the channels through which closings could affect application decisions, including congestion at neighboring field offices and travel time to neighboring offices. $^{2}$ We use walk-in wait time and application processing time as proxies for congestion; closings result in an average increase of 32 percent (4.4 minutes) in walk-in wait time and 11 percent (3.0 days) in processing time. With respect to travel times, we use calculations from Google Maps to estimate that driving time and public transit time to the nearest open field office increase by about 40 percent (10 minutes in driving time and 36 minutes in public transit time). Using an instrumental

\footnotetext{
${ }^{2}$ Note that the closings do not change who reviews and decides the applicant's case, since these decisions are made at state-level Disability Determination Services offices, rather than at local field offices.
} 
variables framework to decompose the decline in applications into various channels, we find that 72 percent of the reduction in applications is attributable to increased congestion at neighboring offices, 3 percent to increased distance, and 24 percent to the fixed cost of switching field offices. These results are consistent with more local field office contacts occurring by phone rather than in person, since congestion costs affect both modes of communication but transportation costs affect only in-person applicants. When we compare these estimates to the expected value of disability benefits, the estimates imply that potential applicants are willing to pay $\$ 1100$ to avoid increased congestion, $\$ 50$ to avoid greater distance, and $\$ 400$ to avoid switching offices.

In contrast to the Nichols and Zeckhauser (1982) hypothesis, we find that field office closings reduce both productive efficiency and targeting efficiency based on current eligibility standards for disability programs. Moreover, if disability programs are also intended to address economic inequality, our results by socioeconomic status indicate that field office closings exacerbate the very inequality that disability programs are intended to reduce. We use our estimates to conduct a cost-benefit analysis of field office closings. On the cost side, we consider the loss in social welfare from lower disability receipt for deserving applicants and the increased time and effort required to apply for disability. On the benefit side, we consider administrative savings from processing fewer applications and shuttering field offices as well as reductions in application costs and earnings decay for individuals who are discouraged from applying. Using conservative assumptions, we estimate a ratio of social costs to social benefits of field office closings of 5 to 1.

In terms of policy implications, our results indicate that, even in a world of online information and applications, in-person information and assistance still matter for applicants with low education and earnings levels. We find evidence that high-socioeconomic-status applicants strategically rush to submit their applications before the field office closes, and that some switch to the online application after the field office closes. In contrast, low-socioeconomic-status applicants exhibit neither of these behaviors and experience a much larger decline in applications.

The paper proceeds as follows. Section 1 reviews the literature on application costs and provides a conceptual framework to evaluate the effects of closings on targeting efficiency. In Section 2, we describe the institutional context of Social Security field office closings and describe the administrative and programmatic data from the Social Security Administration. Section 3 outlines the empirical strategy and Section 4 presents estimates of the effect of closings on the take-up and targeting of disability programs. In Section 5, we interpret our results on take-up and targeting and analyze the channels through which closings reduce disability applications. Section 6 presents 
welfare calculations and Section 7 concludes.

\section{Literature and Framework}

\section{A Literature and contribution}

In the absence of perfect information about individuals' abilities, the government can target social safety programs using two mechanisms. The first is tagging, in which the government conditions eligibility on observable characteristics like poor health or low income to target groups who are deserving or most in need (Akerlof (1978)). In the context of disability programs, the government uses disability screening to limit benefits to those with a disability tag.

In this paper, we study the second type of targeting mechanism: self-screening, in which program rules and application requirements affect what types of individuals decide to apply for benefits. In particular, we study whether hassles associated with using benefits or services - often called "ordeals" in the public finance literature -improve or worsen targeting. Several papers build the theoretical foundation for the effect of hassles on selection, including arguments for queuing (Nichols, Smolensky and Tideman (1971)), work requirements or activities with some disutility (Besley and Coate (1992)), and asset tests (Golosov and Tsyvinski (2006)) as screening devices. Nichols and Zeckhauser (1982) posit that hassles may improve targeting if they impose a higher relative cost on high-ability individuals compared to low-ability individuals. Thus an optimal transfer program that maximizes social welfare may need to sacrifice productive efficiency - time and effort wasted by applicants on hassles - to improve targeting efficiency.

Kleven and Kopczuk (2011) develop a theoretical framework that is particularly relevant for the disability context. They consider a targeted program that uses a monitoring technology involving substantial information collection and complexity (e.g., the disability determination process) to determine whether an individual is deserving. A more complex monitoring technology reduces the likelihood of allowing non-disabled individuals (Type II error) or rejecting disabled individuals who apply ("Type Ib" error, in their terminology), but increases the likelihood of discouraging disabled individuals from applying ("Type Ia" error). They conclude that optimal programs have a high degree of complexity, incomplete take-up, and both Type I and Type II errors.

The question of whether hassles improve or worsen targeting is ultimately an empirical one, and likely depends on the type of hassle and the characteristics of the marginal population. Bertrand et al. (2004) hypothesize that administrative hassles associated with program enrollment may in 
fact deter the individuals that society would like to target to be on these programs. For example, low-ability individuals may face information costs or mental barriers that magnify the effect of application costs on the decision to apply for benefits.

Previous papers have estimated the effect of hassles (or their reduction) on program take-up, but with less attention to the question of targeting (see Currie (2004) for a review). Bettinger, Terry Long, Oreopoulos and Sanbonmatsu (2012) estimate that Free Application for Federal Student Aid (FAFSA) assistance combined with information on financial aid increases college completion by 8 percentage points, or 29 percent, while information alone has no effect. Automatic enrollment, which changes the default to participation, has been shown to increase participation in retirement savings programs dramatically (Madrian and Shea (2001)). Rossin-Slater (2013) finds that the opening of a Women, Infants, and Children (WIC) program office increases the likelihood that pregnant women in surrounding areas use WIC benefits by 6 percent, with effects driven by urban areas. Kopczuk and Pop-Eleches (2007) estimate moderate effects of the availability of electronic filing on take-up of EITC benefits, while Ebenstein and Stange (2010) find no effect of electronic claiming on UI take-up.

Recent papers in the developing world address the targeting question more directly. Cohen, Dupas and Schaner (2015) find in a field experiment in Kenya that increasing the subsidy for antimalarial drugs increases the receipt of drugs but worsens targeting, with one-half of pills going to individuals without malaria. Alatas, Banerjee, Hanna, Olken, Purnamasari and Wai-Poi (2016), which is the closest paper to ours, conduct a field experiment of requiring households in Indonesia to apply for a welfare program in person, rather than the status quo policy of automatic enrollment. Alatas et al. (2016) find that imposing the active enrollment requirement improves targeting efficiency by disproportionately screening out higher-income households. In contrast, we find that application costs reduce targeting efficiency in the US disability context, with the largest effects on applicants with low socioeconomic status and moderately severe conditions. Of course, there are important differences between our US disability context and the Alatas et al. (2016) Indonesia welfare context. One is a difference in the nature of the variation in application costs: we study an increase in application costs from high to even higher, while Alatas et al. (2016) study an increase from zero application cost (automatic enrollment) to low costs. Another difference is that US disability applicants have the option of applying online or by phone rather than showing up at an office in person, while treatment households in Alatas et al. (2016) were required to show up in person. 
This paper makes two contributions to the literature. First, this is the first paper of which we are aware to estimate the effect of application costs on the targeting efficiency of disability programs, a context in which the application process matters critically for targeting because the disability tag is difficult to observe. Second, this paper brings together for the first time detailed administrative data on applicants and specific features of field offices, allowing us to go beyond take-up and study both targeting efficiency and the channels through which closings discourage applicants. To examine targeting efficiency, we use applicant characteristics such as disability type and severity, pre-application earnings, age, education, and language spoken. To study the channels through which closings discourage applications, we collect from SSA program offices several sources of data that have not previously been used for research. These include field office wait times, processing times, and staff counts, which allow us to quantify congestion at neighboring offices; and call volumes to the 800 information line, which shed light on the role of field offices in providing program information. We also calculate driving and public transportation times to field offices using Google Maps software.

\section{B Targeting efficiency framework}

Our goal is to estimate the effect of an increase in application costs on the targeting efficiency of disability programs and on social welfare. We define an improvement in targeting efficiency as follows: when application costs increase from $\eta$ to $\eta^{\prime}>\eta$, targeting efficiency increases if and only if

$$
\operatorname{Pr}\left(R \mid A, \eta^{\prime}\right)>\operatorname{Pr}(R \mid A, \eta)
$$

where $\operatorname{Pr}(R \mid A, \eta)$ is the probability of receiving benefits conditional on applying for benefits at application cost $\eta$. The intuition behind this definition is that, assuming no change in adjudicator standards, the probability of acceptance increases when the applicant pool becomes more deserving. If $\operatorname{Pr}(R \mid A, \eta) \neq 0$, we can rewrite our definition of an improvement in targeting efficiency in terms of the empirical parameters that we estimate:

$$
1<\frac{\operatorname{Pr}\left(R \mid A, \eta^{\prime}\right)}{\operatorname{Pr}(R \mid A, \eta)}=\frac{\frac{\operatorname{Pr}\left(R \mid \eta^{\prime}\right)}{\operatorname{Pr}\left(A \mid \eta^{\prime}\right)}}{\frac{\operatorname{Pr}(R \mid \eta)}{\operatorname{Pr}(A \mid \eta)}}=\frac{\frac{\operatorname{Pr}\left(R \mid \eta^{\prime}\right)}{\operatorname{Pr}(R \mid \eta)}}{\frac{\operatorname{Pr}\left(A \mid \eta^{\prime}\right)}{\operatorname{Pr}(A \mid \eta)}}=\frac{\Delta_{R}+1}{\Delta_{A}+1}
$$

where $\Delta_{R} \equiv \frac{\operatorname{Pr}\left(R \mid \eta^{\prime}\right)-\operatorname{Pr}(R \mid \eta)}{\operatorname{Pr}(R \mid \eta)}$ is the percent change in the number of disability recipients resulting from the closing and $\Delta_{A} \equiv \frac{\operatorname{Pr}\left(A \mid \eta^{\prime}\right)-\operatorname{Pr}(A \mid \eta)}{\operatorname{Pr}(A \mid \eta)}$ is the percent change in the number of disability ap- 
plicants resulting from the closing. When a field office closes, targeting efficiency improves if the percent decline in disability receipt is less than the percent decline in disability applications. Note that this definition assumes that the adjudicator's preferences for who is deserving or undeserving reflects societal preferences. Since societal preferences may differ from the preferences of the official adjudication process, we also present changes in observable characteristics of applicants and recipients, including severity, disability type, education, pre-application earnings, and age. In Section 6, we present a framework to calculate the change in social welfare from field office closings.

\section{Institutional context and data}

\section{A Institutional context}

The Social Security Administration administers the Social Security Disability Insurance (SSDI) and Supplemental Security Income (SSI) programs. SSDI and SSI have the same medical requirements but different non-medical requirements: SSDI requires a work history, while SSI requires low income and assets. Individuals can apply for and receive benefits from both programs concurrently if they meet the requirements of both, with the SSI benefit reduced by the amount of the SSDI benefit.

Potential applicants can apply for SSDI and SSI by filing a claim in person at a Social Security field office, filing a claim over the phone with a claimants' representative at a Social Security field office, or - for SSDI applicants only — by filing the claim online. ${ }^{3}$ Regardless of how the application is filed, the application is generally processed by the field office that serves the ZIP code in which the applicant resides. The applications in our data are identified by the claimant's ZIP code of residence. In processing the claim, the field office verifies that applicants meet the non-medical requirements (work history for SSDI and income and assets for SSI) and often collects information that the disability examiner needs to make a medical decision, such as medical records and (for children) school records. The field office then transfers the application to the state disability determination services (DDS) office, where a disability examiner decides whether the applicant meets medical requirements. Note that field offices do not make decisions about an applicant's case. Applicants can appeal the initial examiner's decision, first to the DDS office itself (in all but 10 states), then to an administrative law judge (ALJ), then to the Appeals Council, and finally, for a very small fraction of cases, to federal court.

The first Social Security field office opened in Austin, Texas, in October 1936. Today there are

\footnotetext{
${ }^{3}$ The SSI application became available only in April 2017, after our study period ends.
} 
approximately 1,230 Social Security field offices in the United States. Field offices serve many functions, including taking applications for new or replacement Social Security cards, providing benefit verifications, assisting with disability and retirement claims, and processing disability claims before transferring them to the state DDS office. However, disability claims take up a disproportionately large amount of staff time, with two-thirds of SSA's administrative budget going to disability claims. ${ }^{4}$ According to SSA testimony, "disability claims...are particularly time intensive as employees help claimants complete detailed forms about medications, treatment, medical testing, work history, and daily activities." 5

Since Social Security field offices provide assistance with applications, the closing of field offices is expected to increase (weakly) the cost of applying. Potential applicants must travel farther for in-person assistance, may experience congestion at neighboring offices, and may find it more costly to gather program information. We use recent Social Security field office closings to study the effect of application costs on selection into disability programs. Although there were very few closings prior to 2000, there have been 118 closings since that year, with approximately half of those closings occurring since 2009 (see Figure 1). The obvious concern with this empirical strategy is that SSA may be closing offices in areas where disability applications are already falling or where the composition of disability applicants is already changing. ${ }^{6}$ To address this issue, we use areas that experience a closing in the future as controls for areas that experience a closing today. The identifying assumption is that the exact timing of the closing is uncorrelated with changes in the number and type of disability applicants. We demonstrate that there are no pre-trends in the outcome variables, that macroeconomic variables such as population and unemployment rate do not exhibit a break at the time of the closing, and that no observable characteristics of the office or location predict the timing of closings.

\footnotetext{
${ }^{4}$ Testimony of Jo Anne B. Barnhart, Commissioner of the Social Security Administration, to the U.S. House of Representatives, March 4, 2003. SSA's administrative budget reflects both field office and state DDS costs. Social Security field office functions include issuing new or replacement Social Security cards, assisting with retirement and disability claims, providing verification of benefits, processing claims, assisting state offices with continuing disability reviews, and conducting SSI non-medical redeterminations.

${ }^{5}$ Testimony of Nancy Berryhill, Deputy Commissioner for Operations, SSA, to Special Committee on Aging, United States Senate, June 18, 2014.

${ }^{6}$ According to a Congressional report, the 64 closings that have occurred since 2009 have been in response to "technological, demographic, and budgetary" changes at the federal level. We show in Appendix Table A.12 that smaller local populations, fewer applications and more offices in close proximity predict a higher likelihood of an office closing, which suggests that the closings themselves are not as good as random. In Section 4.D, we find that our estimates are robust to using unaffected ZIPs as the control group and to using an event study design.
} 


\section{B Data}

We use confidential administrative and program data from the Social Security Administration. We collect data on Social Security field offices from several SSA program offices. From the Office of Analysis, Development, and Support (OADS) and the Office of Earnings, Enumeration, and Administrative Systems (OEEAS), we have data identifying all field offices ever in operation, including field office number, street address, and closing date if it closed (no opening date). From the Office of Public Service and Operations Support (OPSOS), we have data on walk-in wait times at Social Security field offices going back to Fiscal Year (FY) 2005. These wait times are not specific to disability applicants; they reflect the average time that any individual entering a field office waits until being served by a field office worker, and we use them as a measure of field office congestion. We also have data on the number of staff members at each field office going back to FY 1997 from OPSOS, and on Social Security card issuances by field office going back to FY 2005 from OEEAS. Finally, from SSA's Office of Customer Service, we have the volume of calls to SSA's 800 phone number by area code by month from January 2014 to April 2016.

We use data on disability applicants and recipients from a number of sources. We start with 831 application data for all cases with a disability examiner decision between 1990 and 2015. The 831 files report applicant characteristics, including age, body system code (i.e., general disability category), medical diary reason (a measure of severity), and education (for adults only). The 831 files also provide the date on which the application was filed, the date on which the field office transferred the file to a state DDS office, whether the case was allowed at the disability examiner level, and applicant ZIP code up to 2010. For additional applicant characteristics and applicant ZIP codes after 2010, we use data from the Structured Data Repository (SDR), which starts in 2005. Applicant characteristics in the SDR include whether the applicant files online, whether the applicant has legal representation, whether the applicant has a representative payee, whether the applicant has an email address, and whether the applicant speaks English. We use the Disability Research File $(\mathrm{DRF})$ and the Master Earnings File for pre-application earnings of applicants. Finally, we use the Master Beneficiary Record and Supplemental Security Record to observe the final determination for each case at the end of the adjudication process.

We collapse the Social Security data by the ZIP of the applicant's address and link it to publicly available ZIP Code Tabulation Area data from the Census Bureau. We have a total of 33,649 ZIPs; Figure 2 shows their boundaries. For each ZIP code, we use the GIS software to find its centroid and 
apply the Haversine formula to calculate the great-circle distance - the shortest distance over the earth's surface - between ZIP code centroid and each field office in the United States. In addition to this "as-the-crow-flies" distance, we also compute driving distance, driving time, and public transportation time using Google Maps APIs. Combining the distance and time measures with the information on field office closings provided by the SSA, we assign each ZIP to its nearest, second nearest, and third nearest field offices for each quarter from 1990 to 2015. We classify ZIPs into three categories: ZIPs whose nearest office was closed ("closing" ZIPs), ZIPs whose nearest office is the second or the third nearest field office of a closing ZIP prior to the closing event ("neighboring" ZIPs), and all remaining ZIPs ("unaffected" ZIPs). Figure 2 shows the locations of all Social Security field offices since 2000 and demonstrates the classification of closing, neighboring, and unaffected ZIPs. Appendix Figure A.9 shows a zoomed-in version of this map for the state of New York.

We collect ZIP code level demographic information from the 2000 Census and the American Community Survey. Since information at the ZIP level is limited and is not available between Census years, we also collect county level information and link ZIP codes to counties with the largest shared areas. At the county level, we have quarterly data on employment, unemployment, labor force and payrolls from the Bureau of Labor Statistics; semiannual data on broadband access from the Federal Communications Commissions; annual data on personal income from the Bureau of Economic Analysis; annual data on population estimates and business patterns from the Census; and annual data on SSDI/SSI recipients from publicly available SSA publications. Finally, to analyze call volumes to SSA's 800 number, we also link ZIP codes to their respective area codes as of May 2016 using ZIP Express software.

\section{Empirical strategy}

We estimate the effects of field office closings by comparing the number and characteristics of disability applicants and recipients in areas that experience a closing at a given time relative to areas that experience a closing in the future. The motivation for this empirical strategy is that areas that experience closings at some time are likely more similar to each other than they are to areas that never experience a closing. Table 1 presents the characteristics of ZIP codes that have an average of at least three disability applications per quarter in the year 2000, across closing, neighboring and unaffected ZIPs, as defined in Section 2.B. The ZIP means across the three groups are similar; the most apparent differences are that closing and neighboring ZIPs have larger populations and 
more disability applications in the year 2000 than unaffected ZIPs. Given the large number of ZIPs, however, the differences across them are precisely estimated and t-tests find significant differences across the groups for many characteristics. ${ }^{7}$

Given potential differences in observable and unobservable characteristics between closing and unaffected ZIPs, we restrict our sample only to closing ZIPs. For any given closing, we take ZIPs that experience the current closing as treated ZIPs, and ZIPs that experience a closing in the future as control ZIPs. Specifically, we construct our sample as follows. First, we create separate datasets for each of the 118 closings. In each dataset, ZIPs that experience the current closing are labelled as treated ZIPs, while ZIPs that experience a closing more than two years in the future are labelled as control ZIPs. Event quarters are specified relative to the quarter of the closing. Second, to eliminate ZIPs with tiny populations, we drop ZIPs (both treatment and control) with an average of fewer than three disability applications per quarter in the year before the closing. Third, we append all 118 datasets into one dataset.

Figure 3 shows raw plots of the number of disability applications in control and treatment ZIP codes relative to the quarter of the closing. The drop in applications in treatment ZIPs after the closing is apparent, while control ZIPs follow a smooth upward trend in applications. Appendix Table A.8 compares pre-closing characteristics of treatment and control ZIPs and shows that they are similar on demographics, but treatment ZIPs have higher walk-in wait times and more disability applications in the year before closing.

To estimate the effects of the closings in regression form, we estimate the following equation on the sample:

$$
Y_{i s c t}=\alpha_{i}+\gamma_{s t}+\Sigma_{\tau} D_{c t}^{\tau}+\Sigma_{\tau} \delta_{\tau}\left(\text { Treated }_{i c} \times D_{c t}^{\tau}\right)+\epsilon_{i s c t}
$$

where $Y_{i s c t}$ is an outcome (e.g., number of disability applicants) for ZIP $i$ in state $s$ for closing $c$ in quarter $t$. The $\alpha_{i}$ are ZIP fixed effects, and $\gamma_{s t}$ are calendar quarter by state fixed effects. The

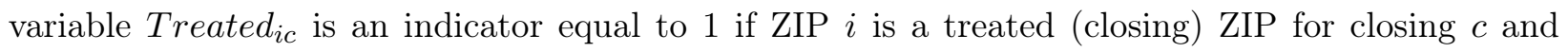
the $D_{c t}^{\tau}$ are indicators equal to 1 if quarter $t$ is $\tau$ quarters after (or before, if negative) the quarter of the closing and 0 otherwise. We weight ZIPs by the number of pre-closing applications, and we cluster standard errors at the closing level. The coefficients of interest are the $\delta_{\tau}$; they represent the difference between treated and control ZIPs in outcome $Y, \tau$ quarters after the closing. The

\footnotetext{
${ }^{7}$ Appendix Table A.7 presents the same summary statistics for all ZIPs in the United States.
} 
graphs presented in the following sections plot the $\delta_{\tau}$ estimates in event time.

For table estimates, we estimate a pre-post version of equation (1):

$$
Y_{i s c t}=\alpha_{i}+\gamma_{s t}+\Sigma_{\tau} D_{c t}^{\tau}+\beta\left(\text { Treated }_{i c} \times \text { Post }_{c t}\right)+\kappa\left(\text { Treated }_{i c} \times \text { Zero }_{c t}\right)+\epsilon_{i s c t}
$$

where Post st $_{\text {is }}$ an indicator equal to 1 if quarter $t$ is after the closing and Zeroct is an indicator equal to 1 if quarter $t$ is the quarter of the closing. We dummy out the quarter of the closing because the closing could occur at the beginning or the end of the quarter, and therefore it is unclear whether to group the quarter of the closing with the "pre" or "post" period. We report estimates of $\beta$ in our tables.

This form of difference-in-differences uses variation in the timing of closings, rather than variation in the occurrence of closings (Guryan (2004); Fadlon and Nielsen (2015)). We use ZIPs that experience a field office closing more than two years in the future as controls for field offices that experience a closing today. The identifying assumption of the difference-in-differences model is that, in the absence of the closing, the number and characteristics of disability applicants and recipients would have evolved similarly in areas that experience a closing today relative to areas that experience a closing in the future. Rather than the closings themselves being random events, the empirical strategy of using future closing ZIPs as controls requires only that the timing of the closings be as good as random. Indeed, in Section 4.D, we find that observable characteristics of field offices predict the likelihood of closing, but not the timing of closings conditional on closing. In the figures presented below, we demonstrate that the treated and control ZIPs exhibit parallel trends in the quarters before the closing in both number of applications and characteristics of disability applicants. In robustness checks, we find similar estimates of the treatment effect using an event study design, but the event study design has pre-trends.

\section{Estimates of the effect of closings on applicants and recipients}

\section{A Effect of closings on take-up in closing ZIPs}

Figure 4 shows the effect of field office closings on the log number of disability applications in closing ZIPs, based on estimates from equation (1), where applications are assigned to quarter based on the date the application was filed. Notice that the treated and control ZIPs exhibit parallel trends in disability applications prior to event quarter 0 . Disability applications fall by 11 percent as a result 
of a field office closing in closing ZIPs (Table 2), and the fall in levels is 12 applications per ZIP per quarter (Appendix Table A.10). It takes two quarters after the closing for disability applications to reach a stable 11 percent decline, likely because some applicants who visited the field office before the closing submit their applications after the closing. The effect is persistent even two years after the closing. Although we cannot test for intertemporal substitution because we cannot identify individuals who do not apply, the persistence of the effects suggests that applicants discouraged by the closing do not apply for at least another two years.

The decline in applications has different implications depending on whether it leads to a decline

in the number of recipients. Figure 4 shows that the number of disability recipients declines by 13 percent in closing ZIPs (Table 3), with allowances still assigned to quarter based on application date. This estimate is statistically different from the 11 percent decline in applications (Appendix Table A.9). The decline in levels is 6 allowances per quarter per ZIP (Appendix Table A.10). The results imply that closings disproportionately discourage applications by those who would have been allowed by SSA adjudicators if they had applied.

\section{B Effect of closings on targeting in closing ZIPs}

Who is screened out by higher application costs? We measure effects on targeting in two ways. First, we estimate the effect of the closings on applicants and recipients separately for each subgroup (first set of columns in Tables 2 and 3) and test for statistical differences across subgroups (see Appendix Table A.9). Second, we estimate the effect of the closings on the proportion of applicants and recipients with a given characteristic (e.g., proportion with mental condition) or on the average value of the characteristic (e.g., average age), similar to the approach taken by Gruber, Levine and Staiger (1999) and Einav, Finkelstein and Cullen (2010) (second set of columns in Tables 2 and 3). While the proportion/average estimates summarize overall effects of the closings on a characteristic, the estimates by subgroup provide a more detailed picture of the effects of the closings. This analysis rests on the assumption, discussed in detail in Section 4.D, that the closings do not affect how applicants are classified.

We find that composition changes are similar at the applicant and recipient levels, so we focus mainly on the applicant level in the exposition, since it provides a direct measure of applicant behavior. We start with measures of health. We categorize applicants into four severity categories: those who are never allowed ("low" severity); those who are denied at the initial level but allowed on appeal ("medium" severity); those allowed at the initial level and labelled "medical improvement 
expected" or "medical improvement possible" ("high" severity); and those allowed at the initial level and labelled "medical improvement not expected" ("very high" severity). ${ }^{8}$ Whether field office closings disproportionately discourage higher severity or lower severity applicants is ex ante ambiguous: higher severity applicants may face higher costs of reaching a neighboring office or applying through other means because of their health, while less severely disabled applicants may no longer find it worth applying given the increase in application costs. As shown in Figure 5, we find that the decline in applications is non-monotonic in severity, with smaller effects for low severity (8 percent) and very-high severity (5 percent) applicants, and larger effects for medium severity (27 percent) and high severity (16 percent) applicants. The differences across severity subgroups are statistically significant.

Another observable measure of health is disability type. We categorize applicants into three disability types based on the body system code on their record: mental conditions, which have accounted for a substantial increase in disability enrollment for both adults and children; musculoskeletal conditions (such as back pain), which have also risen substantially for adults in recent decades; and other physical conditions. ${ }^{9}$ The decline in applications is nearly twice as large for mental conditions (12 percent) and physical conditions (12 percent) compared to musculoskeletal (7 percent) conditions, and this difference is statistically significant.

Turning to socioeconomic status, we estimate the effects of the closings by education, preapplication earnings, and whether the applicant speaks English. We observe these characteristics for adults only and therefore estimate effects on these characteristics excluding SSI children. The effects of the closing are monotonically decreasing in education level: from Figure 5 and Table 2, applications decline by 13 percent for high school dropouts, by 9 percent for high school graduates, and by 4 percent for college graduates, though these differences are not significant. The effects of the closings are also monotonically decreasing in pre-application earnings, which we measure as annual earnings in the five years prior to the year of application. Applications decline by 13 percent in the lowest earnings category $(\$ 0-\$ 5,000)$ but do not change for the highest earnings category (above $\$ 25,000)$. The result of these differential effects is that average annual pre-application earnings

\footnotetext{
${ }^{8}$ SSA's standard for "medical improvement not expected" is as follows: "Medical impairment is extremely severe, as determined on the basis of existing medical technology and/or our experience in administering disability programs. These impairments do not improve over time, and more likely are progressive either by themselves or by reason of related complications. The likelihood of medical improvement so as to permit the individual to engage in substantial gainful activity is extremely remote" (SSA Program Operations Manual System DI 13005.022).

${ }^{9}$ The "other physical" category includes the following body system codes: special senses and speech, respiratory, cardiovascular, digestive, genitourinary, hematological, skin, endocrine, congenital, neurological, cancer, immune system, growth impairment, and special/other.
} 
increase by a statistically significant $\$ 650$, or 4.3 percent, after a closing. Interestingly, Figure 5 shows a spike in applications by the highest-earning applicants in the quarters around the closing. We interpret this spike as anticipatory behavior: higher-earning applicants, who may be more informed, rush to get help from the field office before the office closes. The subsequent decline in applications for this group could reflect intertemporal substitution rather than a true decrease in the number of applications. In contrast, applications from lower-earning applicants remain depressed at the end of the two-year period. Finally, we find that field office closings disproportionately discourage English speakers from applying (8 percent decline) relative to non-English speakers (0.2 percent increase). We find little heterogeneity by age for adults.

We can also present these estimates in absolute numbers, as we do in Appendix Table A.10. Taking the point estimates at face value, we find that for every 10 low-severity potential applicants who are discouraged from applying due to a closing, the closing also discourages 6 medium-severity applicants, 5 high-severity applicants, and 1 very-high-severity applicant. Similarly, for every 10 college graduates discouraged from applying, 631 high school dropouts and 707 high school graduates are discouraged. ${ }^{10}$

All disability programs experience substantial declines in the number of applicants, but the point estimates for the adult SSI (15 percent) and child SSI (15 percent) programs are twice as large as those for the adult SSDI (7 percent) program (Appendix Table A.18). The smaller decline in SSDI applications is consistent with the availability of an online application for SSDI and the higher socioeconomic status of the SSDI population, which might afford easier access to alternatives to the closed field office. ${ }^{11}$

Appendix Figure A.11 depicts effects by subgroup at the recipient level. Changes in age, education, and earnings after the closing are similar in percentage terms for disability recipients and disability applicants. Because severity is defined according to the allowance decision, the severity results are mechanically the same at the applicant and recipient margins.

\footnotetext{
${ }^{10}$ Of course, characteristics of applicants are not necessarily independent; for example, a highly educated potential applicant (considered less deserving on the basis of socioeconomic status) may be more likely to be severely disabled. Indeed, the correlation between college education and very high severity is a positive 0.05. For this reason, we also estimate in Appendix Table A.11 the effect of field office closings on characteristics jointly. We find results that are consistent with the separate education and severity estimates: the effects are largest for potential applicants with lower education levels and medium- and high-severity conditions. College graduates do not experience large declines in any severity category, and similarly, very high severity potential applicants experience small declines regardless of education category. Effects by education-by-disability type cells are also consistent with the separate education and disability type estimates.

${ }^{11}$ We also estimate the effects of field office closings on Social Security card issuances and find null effects, as shown in Appendix Figure A.10.
} 


\section{C Effect of closings in neighboring ZIPs}

We also examine the effects of the closing on neighboring ZIPs, which are ZIPs whose nearest office is the second or third closest office of a closing ZIP prior to the closing event. Neighboring field offices may experience an increase in congestion due to an expanded service area after the closing, which could discourage applications in neighboring ZIPs; indeed, we find evidence for congestion at neighboring offices in Section 5.C. To estimate effects on neighboring ZIPs, we construct the sample in a similar way as the main analysis, except that for each closing the treatment group is neighboring ZIPs for that closing and the control group is neighboring ZIPs in future closings. We estimate equations analogous to (1) and (2), replacing the Treated $_{i c}$ indicator with a TreatedNbr $r_{i c}$ indicator that is equal to 1 if ZIP $i$ is a neighboring ZIP for closing $c$ and 0 otherwise. We find large and persistent decreases in the number of applications and recipients for neighboring ZIPs. The number of applications falls by 5.4 percent and the number of recipients falls by 9.0 percent (Table 4). As shown in Appendix Figure A.12, the control and treatment groups exhibit parallel trends prior to the closing, but applications and allowances fall for the treatment group after the closing and remain depressed two years after the closing.

\section{D Robustness}

The identifying assumption of the difference-in-differences design is that control and treatment ZIPs would experience parallel trends in outcomes in the absence of the field office closing. As seen in Figures 4 and 5, control and treatment ZIPs exhibit parallel trends in the number and composition of applicants prior to the closing. However, it is still possible that the closing itself is prompted by a change in macroeconomic conditions in the treatment ZIPs, and those changes in economic conditions could lead to changes in the number and composition of residents in those ZIP codes. For example, the Social Security Administration may decide to close offices in areas that experience an adverse economic shock or a declining population. In this case, we would mistakenly attribute the change in the number and composition of disability applicants and recipients to the closing, when the closing is merely a symptom of (or coincidental with) changes in macroeconomic conditions.

To probe this threat to validity, we estimate differential trends in macroeconomic conditions between control and treatment ZIPs. Specifically, we put macroeconomic variables on the lefthand side of equation (1) and plot estimates of the $\delta_{\tau}$ coefficients in Appendix Figure A.13. We find smooth trends through the closing date in population, labor force, unemployment rate, and personal 
income. Graphs show a positive trend in population, which suggests that the decline in applications is not explained by outmigration from the areas surrounding the closed office. The other graphs show positive trends in economic conditions: increasing labor force and personal income, and declining unemployment rates. However, there is no trend break in any of the macroeconomic variables; the trends are gradual and are not consistent with the abrupt drop in disability applications and receipt in treated ZIPs. These figures suggest that the changes in the number and composition of applicants and recipients are not caused by macroeconomic shocks. We also augment equations (1) and (2) to include controls for the local unemployment rate and population and find no change in the estimates (Appendix Figure A.14). ${ }^{12}$

To probe whether offices were closed strategically based on local conditions, we investigate what observable factors predict 1) the likelihood of closing and 2) the timing of a closing conditional on closing, with results presented in Appendix Table A.12. ${ }^{13}$ Although some factors consistently predict the likelihood of a closing (columns 1-3), no observable characteristic consistently predicts the timing of a closing conditional on closing (columns 4-6).

We also probe the robustness of our results to different measures of distance, since distance determines whether ZIPs are classified as closing, neighboring, or unaffected. Our main results use straight-line distance and define closings ZIPs as ZIPs whose nearest office closes. We estimate the effects of the closing using two other strategies: 1) using driving time as the measure of distance to classify ZIPs, and 2) defining closing ZIPs as ZIPs within a certain radius (as measured by straight-

\footnotetext{
${ }^{12}$ Another potential threat is that control ZIPs (those that experience their own closing at least two years later) could be neighboring ZIPs of the closing for which they serve as a control. Since, as we show, neighboring ZIPs also experience effects from the closing, using neighboring ZIPs as controls would lead us to underestimate the effect of the closing on surrounding areas. Empirically, we find that just 0.3 percent of control ZIPs are neighbors and the estimates do not change when we exclude neighbors from the sample.

${ }^{13}$ According to Congressional testimony, SSA has not considered local economic or other conditions in deciding what offices to close ("Reduction in Face-to-Face Services at the Social Security Administration," United States Senate Special Committee on Aging, Summary of Committee Staff Investigation, No Date). To examine whether local characteristics predict the likelihood of a closing, for each year between 2000 and 2012, we use all open offices in a given year and estimate the following equation:
}

$$
\text { Closing }_{i}=\alpha+\beta_{1}{\text { Pop } 2000_{i}+\beta_{2} \text { Density }_{i}+\beta_{3} \text { Apps }_{i}+\beta_{4} \text { FOProcess }_{i}+\beta_{5} \text { NumOffice }}_{i}+\beta_{6} \text { Wait }_{i}+\epsilon_{i}
$$

where $\operatorname{Pop}_{2000}$ is the population of the service area of office $i$ in the year 2000; Density is the population density of the service area of office $i$ in the year 2000; Apps $s_{i}$ is the number of disability applications submitted in office $i$ 's service area in the year before the closing; FOProcess $i$ is the application processing time for office $i$ in the year before the closing; NumOffice ${ }_{i}$ is the number of offices within 20 kilometers of office $i$ before the closing; and $W a i t_{i}$ is walk-in wait time for office $i$ in the year before the closing (available only for 2006 and later). To examine whether local characteristics predict the timing of closing conditional on closing, for each year between 2000 and 2012 , we limit the sample to offices that are open in that year but will close in the future and estimate the following equation:

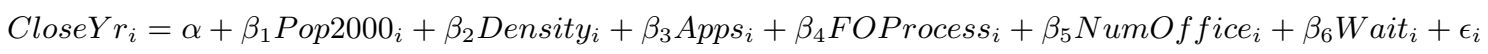

where CloseYri is the year in which office $i$ closed. The results of both are shown in Appendix Table A.12. 
line distance) of the field office. The estimates using the alternative measures are given in Appendix Table A.13 and are within 15 percent of the main estimates for applications and within 6 percent of the main estimates for recipients.

As another robustness check, we estimate the effects of the closings using event study specifications instead of the difference-in-differences approach. We use the following estimating equation:

$$
Y_{i s c t}=\alpha_{i}+\gamma_{s t}+\Sigma_{\tau} \delta_{\tau} D_{c t}^{\tau}+\epsilon_{i s c t}
$$

where we estimate one version that includes unaffected ZIPs as controls and another version that includes only closing ZIPs. For control (unaffected) ZIPs, all $D_{c t}^{\tau}$ are set to zero. For treatment (closing) ZIPs, the $D_{c t}^{\tau}$ are equal to one when the quarter is $\tau$ quarters after (or before, if negative) the closing. Figure 6 shows both versions of the event study. The version using unaffected ZIPs as controls (left side) shows an upward pre-trend in the number of applications, but the fall in applications in treatment ZIPs after the closing is still apparent, and the estimates are approximately the same magnitude (around 10 percent) as in our preferred empirical strategy. The version using only closing ZIPs (right side) has a less pronounced pre-trend and a drop of similar magnitude in applications. In addition, we estimate the effects of the closing using the difference-in-differences specification with different minimum lengths of time between treatment closings and control closings; the estimates using windows of 4, 6, 10, and 12 quarters are nearly indistinguishable from our original estimates using an 8-quarter window (see Appendix Figure A.15).

Finally, the interpretation of our estimates of the effect of closings on the composition of applicants and recipients depends on whether the closings affect the classification of applicants. If, for example, the closings affect the likelihood that an applicant is classified as high severity, then the change in severity composition reflects not only differential responsiveness of severity types to the closing, but also a change in the likelihood of being classified as a given severity type. For severity, the severity classification decision is made at the state DDS office, which does not change after the local field office closes. Within DDS offices, cases are assigned to disability examiners in an effectively random way and not based on geography. ${ }^{14}$ Examiners are responsible for verifying that the medical records in the case are complete, and if incomplete request full medical records. Our back-of-the-envelope calculations indicate that the decline in applications from a field office closing is on average less than 2 percent of the DDS caseload, which makes it unlikely that the

\footnotetext{
${ }^{14}$ See, e.g,. Maestas, Mullen and Strand (2013) for a description of the assignment system.
} 
field office closing has an effect on disability examiner decision-making. For disability type, the examiner also decides this classification (e.g., mental or musculoskeletal) based on what is stated in the application and on the applicant's medical records. It is possible that field office assistance affects the number or type of medical conditions listed and thereby affects severity or disability type classifications, and we cannot test this possibility directly. For socioeconomic status, we measure pre-application earnings using administrative data, so there can be no change in the pre-application earnings classification after the closing. Education level and age are self-reported on the application, but we have no reason to believe that field offices affect how applicants report them. ${ }^{15}$

\section{$5 \quad$ Interpreting the effects of field office closings}

\section{A Interpreting effects on take-up}

We find in the previous section that field office closings reduce disability applications by 7 percent for SSDI and 15 percent for SSI, and reduce disability receipt by 12 percent for SSDI and 15 percent for SSI. These are large effects, with our back-of-the-envelope calculations implying a value of time of approximately $\$ 100$ per hour for both SSI and DI applicants. This implied value of time is consistent with Alatas et al. (2016), whose estimates suggest an implied value of time of about $\$ 20$ per hour in the Indonesian context, where wages are several times lower than in the US. ${ }^{16}$

How do the effects of field office closings compare to the effects of hassles in other contexts? Bettinger et al. (2012) estimate a 29 percent increase in college completion from providing assistance with the FAFSA, while Madrian and Shea (2001) estimate a 130 percent increase in 401(k) enrollment from automatically enrolling individuals. Quasi-experimental estimates of hassle reductions are smaller: Rossin-Slater (2013) finds that openings of WIC offices increase take-up by 6 percent in surrounding areas, Kopczuk and Pop-Eleches (2007) estimate a 12 percent increase in EITC claiming from electronic filing, and Ebenstein and Stange (2010) find no effect of Internetbased UI claiming on take-up. We also compare the effects of the closings to other determinants of

\footnotetext{
${ }^{15}$ This observation is based on our visits to field offices and discussions with current and former field office staff.

${ }^{16}$ To calculate implied value of time in our setting, we assume, conservatively, that those who do not apply because of the closing lose two years of DI benefits, which average $\$ 1,300$ per month. With an overall $2 / 3$ probability of allowance, the expected benefit of applying is $\$ 20,800$. From our estimates, closings reduce the probability of applying by 7 percent for the DI program. If the field office closing increases the amount of time required to apply by 15 hours, then the value of time that rationalizes the decision not to apply is $(0.07 * \$ 20,800) / 15=\$ 97$. By similar logic, and using the 15 percent decline and $\$ 700 /$ month in benefits, the value of time for SSI recipients is $\$ 112$. We calculate an implied value of time in Alatas et al. (2016) as follows. They find a 15 percent decline in the take-up of benefits with an estimated $\$ 700 \mathrm{NPV}$, in response to an estimated half-day increase in travel and wait time. If we assume a 5 hour increase in time to apply, the implied value of time is $\left(0.15^{*} \$ 700\right) / 5=\$ 21$.
} 
disability application and receipt, such as economic conditions, program rules, and health shocks. These comparisons suggest that the closing of a field office has effects at least as large as a 10 percent change in earnings or a 10 percent change in replacement rates, but much smaller than a severe health shock. Of course, the normative implications of application reductions from closings versus earnings gains or health shocks are likely different. ${ }^{17}$

The $\$ 100$ per hour implied value of time is much larger than the monetary opportunity cost of time for low- and medium-wage individuals, especially SSI applicants, who by definition have low income and assets. Why are the effects on take-up so large? There are several potential explanations. First, potential applicants may have difficulty finding alternative sources of assistance after a closing because of credit constraints and legal restrictions. In principle, potential applicants could promise a third party some fraction of their disability benefits if their application is approved, but government regulations restrict compensation to third-party representatives of disability applicants. ${ }^{18}$ Second, potential applicants may exhibit present bias, in which they underweight the large benefits of applying and overweight the additional costs of applying resulting from the closing. Third, the closings may cause potential applicants to update their beliefs about the disability system. After experiencing a closing and resulting inconveniences, potential applicants may adjust their beliefs about the probability of rejection or about the difficulty of interacting with the system in the future.

\section{B Interpreting effects on targeting}

Field office closings present a tradeoff between Type I and Type II error: they discourage applications among the less deserving, but also discourage applications among the more deserving (increasing "Type Ia" error, in the language of Kleven and Kopczuk (2011)). We apply the definition of targeting efficiency based on adjudicator preferences from Section 1.B to our results. Based on the 11 percent decline in applications and 13 percent decline in allowances (statistically different), we find that

\footnotetext{
${ }^{17}$ Black, Daniel and Sanders (2002) study the effects of the coal boom and bust on disability payments. They estimate that a 10 percent increase in earnings reduces DI payments by 3 to 4 percent and SSI payments by 4 to 7 percent. Duggan and Imberman (2009) decompose DI program growth from 1984 to 2003 into various determinants, including program changes and economic conditions. Their estimates suggest that a 10 percent increase in replacement rates would increase DI enrollment by 7 percent. With respect to the effect of health shocks, the Meyer and Mok (2013) estimates suggest that having a chronic severe condition increases the likelihood of disability receipt by 88 percent relative to a chronic non-severe condition.

${ }^{18}$ Federal regulations require that fees are the smaller of 25 percent of past due benefits or the amount of the fee set by SSA (COFR $§ 404.1730)$. Third-party representation is much less common at the initial level than at the appeals level, when applicants receive more "past due benefits" if their case is approved.
} 
field office closings reduce targeting efficiency:

$$
\frac{\Delta_{R}+1}{\Delta_{A}+1}=\frac{-0.13+1}{-0.11+1}<1
$$

If societal preferences differ from adjudicator preferences, then changes in observable characteristics of applicants and recipients are also relevant for assessing targeting efficiency. The effects of the closing on disability applications are decreasing in socioeconomic status, but they are nonmonotonic in severity, with smaller effects for low and very-high severity applicants and larger effects for medium and high severity applicants. Excluding the low severity group, we could explain the results by severity with the hypothesis that the value of the benefits is increasing in severity and the opportunity cost of applying is decreasing in severity. But why do low severity applicants continue to apply? We find evidence that low severity applicants are a highly selected group, being much more likely than the other severity groups to have experienced zero or low earnings in the two years before they apply (see Appendix Table A.14). Therefore we present a model that explains the results by severity considering both a health effect and a selection effect.

Let potential disability applicants differ on two dimensions: health $h \in[0,1]$ where $h=1$ is the best health, and skills $s \in[0,1]$ where $s=1$ is the highest skills. Skills are negatively correlated with health in the potential applicant pool as a result of selection, which we model by making $s$ a function of $h$, with $s^{\prime}(h)<0$. Wages are determined by the function $w(s, h)=\min \{h, s(h)\}$, meaning that poor health will be the binding constraint on wages for the highest severity individuals and skills will be the binding constraint on wages for the lowest severity individuals. Potential disability applicants apply if and only if

$$
\begin{aligned}
p(h) b+ & (1-p(h)) w(s, h)-\eta>w(s, h) \\
& \Longleftrightarrow p(h)[b-w(s, h)]>\eta
\end{aligned}
$$

where $b$ is the amount of disability benefits, $p(h)$ is the likelihood of being approved for benefits, with $p^{\prime}(h)<0$, and $\eta$ is the cost of applying. We assume $b>w(s, h)$.

Let $g(h) \equiv p(h)[b-w(s, h)]$. Consider an individual at the bottom of the health distribution, so that $h<<s$. Then $g(h)=p(h)[b-h]$ and

$$
\frac{\partial g(h)}{\partial h}=p^{\prime}(h)[b-h]-p(h)<0
$$


since $p^{\prime}(h)<0$. The first term reflects that the likelihood of allowance is decreasing in health, and the second term reflects that the opportunity cost of applying is decreasing in health. Thus, when $h$ is small (i.e., going from very high severity to high severity), $g(h)$ is unambiguously decreasing in $h$ and so very high severity types are more likely to continue applying when application costs increase.

Now consider an individual at the top of the health distribution, so that $h>>s$. Then $g(h)=$ $p(h)[b-s(h)]$ and

$$
\frac{\partial g(h)}{\partial h}=p^{\prime}(h)[b-s(h)]-p(h) s^{\prime}(h) \gtrless 0
$$

since $p^{\prime}(h)<0$ and $s^{\prime}(h)<0$. The first term reflects that the likelihood of allowance is decreasing in health, but the second term reflects the selection effect that low severity types are more likely to be low-skilled. Thus, when $h$ is large (i.e., going from medium severity to low severity), $g(h)$ could be increasing in $h$, in which case low severity types will be more likely to continue applying when application costs increase.

Figure 7 provides a visual depiction of the model in which health $h$ in the potential applicant pool has density $f(h)$. The benefit function $g(h)$ is non-monotonic in $h$ because individuals in relatively good health (low $h$ ) are low-skilled and therefore also have a high value of disability benefits. The function $p(h)$ starts at $p(h)=1$ at the very bottom of the health distribution (i.e., very high severity) and is decreasing in $h$. The line $\operatorname{Pr}(R \mid A, \eta)$ indicates the average probability of allowance in the potential applicant pool at baseline, which depends on both $p(h)$ and $f(h)$. In the baseline case, the function $g(h)$ is everywhere above the application cost $\eta$, so all potential applicants apply for benefits. When application costs increase from $\eta$ to $\eta^{\prime}>\eta, g(h)$ is now below $\eta^{\prime}$ for some potential applicants in the middle of the health distribution, and so these medium-severity applicants no longer apply. Intuitively, targeting efficiency improves if the group that was screened out has a lower probability of allowance than the previous applicant pool, and it worsens if the group that was screened out has a higher probability of allowance than the previous applicant pool.

\section{C Channels for closing effects}

Our estimates give the effect of field office closings on the number and composition of disability applicants and recipients. A key question in interpreting these results is through what channels the closings affect disability applications. We use detailed Social Security data on field office features and GIS data to measure the effects of the closing on various channels: congestion at neighboring 
field offices, which could reduce the quantity or quality of assistance received; travel time to the next field office; and other channels, including the costs of acquiring program information and network effects.

Congestion at neighboring offices: Congestion at the neighboring office can take many forms, including longer waiting times to get assistance or a decline in the amount or quality of assistance received. Based on estimating equation (1), Figure 8 shows that the closing causes an increase of 32 percent (4.4 minutes) in walk-in wait time and an increase of 10 percent (3.0 days) in application processing time for closing ZIPs. ${ }^{19}$ Neighboring ZIPs experience similarly large increases in congestion measures (see Table 4 and Appendix Figure A.16). Note that these measures are merely proxies for overall congestion, which can take many forms, including less time for assistance and lower quality of assistance. We expect congestion to affect not only in-person interactions with the field office, but also the larger number of interactions that occur by phone since applicants are generally directed to their local field office phone line for assistance.

Travel times: We use calculations from Google Maps to estimate increases in driving distance, driving time, and public transportation time, using estimating equation (2). We find that the closings result in an increase of about 40 percent in all types of travel cost measures (10 minutes in driving time, 12 kilometers in driving distance, and 36 minutes in public transit time). Unlike the congestion measures, which include behavioral responses, our estimates for changes in travel time and distance are purely mechanical; we do not use actual trips of potential applicants to estimate them. However, the mechanical estimates provide a proxy for the increase in travel costs and we use them in the interpretation of our results. We expect travel times to affect only potential applicants who visit an office in person. We find that the effects of closings are decreasing in distance to the closest (closed) office; the effects are non-monotonic in distance to the neighboring office, but effects are largest when the neighboring office is very far (Appendix Table A.15).

Other channels: Another potential mechanism for the effect of closings on applications is the cost of acquiring program information. According to SSA officials, field offices stopped doing

\footnotetext{
${ }^{19}$ We also estimate effects on the number of field office staff per capita in the service area of the ZIP's nearest field office. We find that the number of staff per capita actually increases (by 30 percent) after a closing, which is consistent with SSA's policy of reassigning staff from the closed office to nearby offices. However, staff count is only one input into field office congestion; closings may affect staff productivity as reassigned staff learn new procedures or develop new relationships with schools and health care providers. In addition, depending on their location, offices often face much higher demand for DI services than SSI services, or vice versa, and may therefore employ field office staff who specialize in one of the programs. When staff who specialize in one program transfer to an office with high demand for the other program, it may take time for the transferred staff to learn the details of the other program. We use walk-in wait time and field office processing time to measure congestion because they are direct measures rather than inputs.
} 
community outreach about SSA programs in the early 2000s due to budget cuts, so the role of field offices in providing program information is limited to individuals who visit the field office. Although we do not have direct measures of information acquisition costs, we find evidence, shown in Appendix Figure A.17, that the closings stem a downward trend in call volumes to SSA's 800 number, which handles inquiries regarding disability applications and other Social Security matters. ${ }^{20}$ This suggests that field offices, when open, provide information about SSA programs. ${ }^{21}$ Closings may also affect applications through network effects; for example, closings may discourage one person from applying and that person may affect the application decisions of others through network effects (Bertrand, Luttmer and Mullainathan (2000); Dahl, Kostol and Mogstad (2014)). If this mechanism were important in the field office context, we might expect to see the effect of field office closings increasing over time. Instead, from Figure 4, we see that the number of applications declines in the first few quarters and then remains at a lower level for the rest of the two-year window.

Decomposition of channels: We use an instrumental variables framework to decompose the decline in applications into three channels: congestion at the neighboring office, travel time, and the fixed cost of switching offices. The fixed cost of switching offices could include several components: the effect of physical proximity to an SSA office (e.g., potential applicants inquire about benefits because they see the SSA sign); effort to figure out where the next office is and how to get there; the destruction of relationships between the field office and local health care providers or schools; match quality between the field office staff and local population (e.g., on race or other demographics); and updating by potential applicants about the likelihood of rejection or the difficulty of interacting with the system in the future. The structural equation of interest is the following:

$$
Y_{i s c t}=\alpha_{i}+\gamma_{s t}+\beta \text { Congestion }_{i c t}+\kappa \text { Distance }_{i c t}+\delta N e w O \text { fice }_{i c t}+\epsilon_{i s c t}
$$

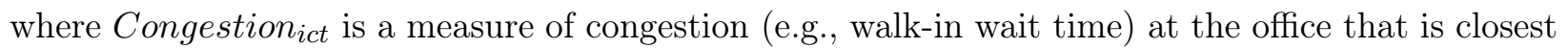

\footnotetext{
${ }^{20}$ We have call volumes by area code by month from January 2014 to April 2016 from SSA's Office of Telephone Services. We estimate an event-study-style regression using the 15 field office closings that occur in 2014:

$$
Y_{i t}=\alpha_{i}+\Sigma_{\tau} \beta_{\tau} D_{i t}^{\tau}+\mu_{t}+\epsilon_{i t}
$$

where $Y_{i t}$ is call volume from area code $i$ in month $t ; \alpha_{i}$ are area code fixed effects; and $\mu_{t}$ are calendar month fixed effects. The vector $D_{i t}^{\tau}$ includes indicator variables for each of the months before and after a closing. The sample includes all area codes in the United States, but the $D_{i t}^{\tau}$ are set equal to zero for unaffected area codes; the unaffected area codes help to identify the $\mu_{t}$. Unfortunately, the pre-period is limited because all but one of the 15 closings occurs in March of 2014, just two months after the data begin. Although the pre-period is limited, we find evidence that closings stem a downward trend in call volumes to the 800 number.

${ }^{21}$ In Appendix Table A.16, we estimate effects with respect to measures of information, such as the proportion of the area receiving or applying for disability, the Chetty, Friedman and Saez (2013) EITC information measure, and broadband access.
} 
to ZIP $i$ in quarter $t$, Distance $_{i c t}$ is the driving distance between ZIP $i$ and its closest office in quarter $t$, and NewOffice ict $_{\text {is }}$ an indicator for whether ZIP $i$ has a different closest office than it did earlier in the sample period (equivalent to Treated $_{i c} \times$ Post $_{t}$ ). The NewOffice $i c t$ variable, which captures the fixed cost of switching offices, reflects effects of the closing not captured by congestion and distance.

These variables could be endogenous to the number of disability applications; for example, a rural ZIP may have longer distances than an urban ZIP and also more disability applications because of a less healthy population. For this reason, we use instruments that limit the variation in each endogenous variable to that induced by 1) the closing itself and 2) differences in wait time (or distance) between the closed office and now-closest (neighboring) office for closing ZIPs. The first stage equations are as follows:

$$
\begin{gathered}
\text { Congestion }_{\text {isct }}=\alpha_{i}+\gamma_{s t}+\beta_{1}\left(\text { Treated }_{i c} \times \text { Post }_{t} \times W \text { Dif }_{i c}\right)+\beta_{2}\left(\text { TreatedNbr }_{i c} \times \text { Post }_{t}\right)+\nu_{\text {isct }} \\
\text { Distance }_{\text {isct }}=\alpha_{i}+\gamma_{s t}+\kappa_{1}\left(\text { Treated }_{i c} \times \text { Post }_{t} \times D D i f f_{i c}\right)+\xi_{\text {isct }}
\end{gathered}
$$

where $W D i f f_{i s c}$ is the difference between the walk-in wait time at the now-closest office and the walk-in wait time at the previously-closest office in the four quarters before the closing; Treated $b r_{i c} \times$ Post $_{t}$ is an indicator equal to 1 if ZIP $i$ is a neighboring ZIP of closing $c$ after the closing; and $D D i f f_{i c}$ is the difference between the driving distance from ZIP $i$ to the now-closest office and the driving distance from ZIP $i$ to the previously-closest office. ${ }^{22}$ By construction, NewOffice ${ }_{i c t} \equiv$ Treated $_{i c} \times$ Post $_{t}$, so we assume that NewOffice ict $_{\text {is }}$ exogenous (i.e., its own instrument).

The first stage, reduced form, OLS, and IV estimates are given in Table 5, with all estimated on the sample of closing and neighboring ZIPs. As expected, the first stage effects are strong and highly significant: a one-minute difference in pre-closing wait times between the closest and secondclosest office predicts a 0.5-minute higher wait-time after the closing; wait times for neighboring ZIPs increase by 4.6 minutes as a result of closings; and a one-kilometer difference in driving distance predicts a one-kilometer increase in driving distance after the closing. The IV estimates in Table 5 indicate that every additional minute of wait time (which proxies for other types of congestion) reduces disability applications by 1.6 percentage points, every additional kilometer of

\footnotetext{
${ }^{22}$ We use the difference in wait times as the instrument instead of just pre-closing wait time at the now-closest office because walk-in wait times are spatially correlated: a high wait time at the now-closest office predicts a high wait time at the previously-closest office, and therefore does a poor job predicting the increase in wait time that the ZIP experiences after the closing. Similarly, we use the difference in distances because distances are spatially correlated: they are longer in rural areas than urban areas.
} 
distance from a field office reduces applications by 0.03 percentage points, and switching to a new office reduces applications by 2.3 percentage points. Scaling these estimates up by the actual changes after the closing, we find that increased congestion accounts for 6.8 percentage points of the decline in applications (72 percent), driving distance for 0.3 percentage points of the decline (3 percent), and the fixed cost of switching offices for 2.3 percentage points of the decline (24 percent). When we compare these estimates to the expected value of benefits, the estimates imply that potential applicants are willing to pay around $\$ 1100$ to avoid increased congestion, $\$ 50$ to avoid greater distance, and $\$ 400$ to avoid switching offices. ${ }^{23}$ Notice that the OLS estimates underestimate the effect of congestion by an order of magnitude and overestimate the effect of office switching, likely because offices that experience higher congestion are in areas with higher demand for disability benefits.

\section{D Substitution to online application and third-party representation}

Resources that provide information and assistance with disability applications may mitigate the effects of field office closings. We study the effects of the closings on the use of two potential field office substitutes: the online application and third-party representation. As shown in Table 2, the number of applicants who file in person or by phone declines by 15 percent, while the number of applicants who file online increases by 7 percent, which could reflect either less discouragement among those who submit online or substitution to the online application. Nearly all of the shift to the online application is driven by college graduates, rather than high school graduates or high school dropouts (see Appendix Table A.17). If we interpret the estimates in terms of applicant behavior, they suggest that more-educated applicants substitute to the online application, while less-educated applicants do not. The closing increases the likelihood of having representation, but the change is not significant, likely because only 5 percent of applicants are represented at the initial level at baseline.

\footnotetext{
${ }^{23}$ We calculate dollar values of each cost in a similar manner to the implied value of time calculations above. We assume that those who do not apply because of the closing lose an average monthly benefit of $\$ 1000$ for two years. With an overall $2 / 3$ probability of allowance, the expected benefit of applying is $\$ 16,000$. From the estimates in Table 5 , congestion reduces the probability of applying by 6.8 percent, driving distance by 0.3 percent, and office switching by 2.3 percent. Multiplying these percentage declines by $\$ 16,000$ yields $\$ 1100, \$ 50$, and $\$ 400$, respectively.
} 


\section{$6 \quad$ Welfare implications of field office closings}

We calculate the change in social welfare resulting from the field office closings using the following framework. Let $b_{r}(n) \equiv b_{1}-c_{1}-c_{2}(n)$ be the net social benefit from an approved disability application given the number of field offices $n$. This term $b_{r}$ includes the value of providing disability benefits to a disabled individual relative to the average taxpayer net of the cost of public funds $\left(b_{1}\right)$, minus the cost of reviewing the application $\left(c_{1}\right)$ and the cost to the applicant of applying $\left(c_{2}\right)$. Let $b_{n}(n) \equiv c_{1}+c_{2}(n)$ be the net social cost from rejecting an application, which includes the cost of reviewing the application $\left(c_{1}\right)$ and the cost to the applicant of applying $\left(c_{2}\right)$. Let $F$ be the administrative cost (i.e., rent) of operating a field office. For a number of recipients $r(n)$ and a number of applicants $a(n)$, the net social benefit of having $n$ field offices is

$$
\begin{aligned}
W(n) & \equiv b_{r}(n) r(n)-b_{n}(n)[a(n)-r(n)]-F n \\
& =\left[b_{1}-c_{1}-c_{2}(n)\right] r(n)-\left[c_{1}+c_{2}(n)\right][a(n)-r(n)]-F n \\
& =b_{1} r(n)-\left[c_{1}+c_{2}(n)\right] a(n)-F n
\end{aligned}
$$

The change in social welfare from reducing the number of offices from $n$ to $n-1$ is

$$
\begin{aligned}
W(n-1)-W(n)= & {\left[b_{1} r(n-1)-\left(c_{1}+c_{2}(n-1)\right) a(n-1)-F(n-1)\right]-\left[b_{1} r(n)-\left(c_{1}+c_{2}(n)\right) a(n)-F n\right] } \\
= & \underbrace{b_{1}[r(n-1)-r(n)]-\left[c_{2}(n-1)-c_{2}(n)\right] a(n-1)}_{\text {costs of closing }} \\
& \underbrace{-c_{1}[a(n-1)-a(n)]-c_{2}(n)[a(n-1)-a(n)]+F}_{\text {benefits of closing }}
\end{aligned}
$$

The costs of the closing include the value of disability benefits to discouraged recipients $\left(b_{1}[r(n-\right.$ 1) $-r(n)]$ ) and the additional application time and effort for applicants who continue to apply $\left(\left[c_{2}(n-1)-c_{2}(n)\right] a(n-1)\right)$. The benefits of the closing include administrative savings from not paying rent on the office $(F)$ and administrative savings from processing fewer applications $\left(c_{1}[a(n-\right.$ 1) $-a(n)])$, including the cost of public funds for both, as well as savings to discouraged applicants from applying $\left(c_{2}(n)[a(n-1)-a(n)]\right) .^{24}$

\footnotetext{
${ }^{24}$ We assume in this analysis that all disability recipients are deserving of benefits and all rejected applicants are undeserving - in other words, no Type I or Type II errors in the disability determination process. To reconcile this assumption with the model presented in Section 5.B in which the probability of allowance is greater than zero for all applicants, we can adjust that model such that applicants observe a subjective measure of health while disability adjudicators use an objective measure of health.
} 
Table 6 presents our cost-benefit analysis estimates, with detailed calculations presented in the Appendix. We start with the costs of the closing. To calculate the value of benefits to recipients, we assume that disability recipients value cash benefits and health insurance 50 percent more than the average taxpayer, reflecting a higher marginal utility of income for a disabled individual, net of the deadweight loss of taxation. We also assume, conservatively, that discouraged applicants lose only two years of disability benefits as a result of the closing. To estimate the cost of increasing applicant time and effort, we calculate the value of lost time from increased travel and wait times and the lost earnings from the increase in processing times based on estimates from Autor et al. (2015). Total costs are around $\$ 19.3$ million per year, with the vast majority of this loss coming from the value of benefits to recipients. Considering losses to non-disability applicant visitors to the field office would increase the cost of the closing further.

Turning to benefits of the closing, we estimate the administrative savings from processing fewer applications by dividing the total annual administrative budget spent on disability programs by the number of applications processed by field offices in a year, and then multiplying this ratio by the reduction in applications. We estimate savings for discouraged applicants who no longer incur application costs. Finally, we take administrative savings from the closing itself from SSA testimony. Total benefits are around $\$ 4.1$ million per year.

Putting these figures together, we find that the social costs of the average field office closing outweigh the social benefits by a ratio of 5 to 1 , mostly because of the large loss in social welfare from discouraging applicants who would have been allowed had they applied. As an alternative analysis, we calculate how much more the average accepted applicant must value disability benefits relative to the average taxpayer for the closings to have no net effect on welfare. We find that if the average discouraged recipient values disability benefits at least 2.6 percent more than the average taxpayer, then the closings are welfare-reducing.

\section{Conclusion}

The effect of application costs on the targeting of social safety net programs is theoretically ambiguous: application costs could improve targeting if they discourage high-ability people with a high opportunity cost of time from applying, or they could worsen targeting if they disproportionately discourage low-ability people from applying. In this paper, we provide the first evidence on this question in the context of disability programs, which are some of the largest social programs 
in the developed world. We find that the closings of Social Security field offices, which provide assistance with disability applications, reduce the number of disability applications by 11 percent and the number of recipients by 13 percent in neighborhoods whose nearest office closes, and have smaller but sizable effects in neighborhoods around neighboring offices. The effects are persistent, with applications showing no sign of recovering even eight quarters after the closings. We also use detailed administrative data on applicant characteristics to determine who is screened out by higher application costs. Closings disproportionately discourage applicants with lower education and pre-application earnings levels and applicants with moderately severe conditions.

What are the policy implications of these results? First, the services provided by field offices are valuable to disability applicants and are instrumental for 11 percent of applicants in the decision to apply. This raises the question of why private industry does not attempt to meet the demand for assistance with disability applications. Possible reasons include credit constraints faced by disability applicants or government regulations that limit the compensation of disability representatives. Second, we find that field office closings affect certain populations more than others. Field office closings appear particularly consequential for potential applicants with low levels of education and earnings, who are less likely to substitute to the online application or strategically submit their application before the office closes. Future decisions about field office placement could consider the distributional consequences of closings. Third, application costs have particular significance in the context of disability programs, since health status is available to the disability agency only if the individual applies. If application costs discourage truly disabled individuals from applying, as we find in this paper, the disability agency has few other ways to identify these individuals and provide them with benefits.

In terms of normative implications, Nichols and Zeckhauser (1982) hypothesize that hassles may increase overall social welfare by sacrificing a small amount of productive efficiency (i.e., more applicant time and effort required to apply) for a large increase in targeting efficiency. We find instead that the increase in hassles induced by Social Security field office closings reduces both productive efficiency and targeting efficiency, as measured by current standards for disability receipt. Moreover, if disability programs are also intended to address economic inequality, then the results by socioeconomic status indicate that field office closings exacerbate the very inequality that disability programs are intended to mitigate. 


\section{References}

Akerlof, George A., "The Economics of "Tagging" as Applied to the Optimal Income Tax, Welfare Programs, and Manpower Planning," American Economic Review, 1978, 68 (1), 8-19.

Alatas, Vivi, Abhijit Banerjee, Rema Hanna, Benjamin A Olken, Ririn Purnamasari, and Matthew Wai-Poi, "Self-Targeting: Evidence from a Field Experiment in Indonesia," Journal of Political Economy, 2016, 124 (2), 371-427.

Autor, David H., Nicole Maestas, Kathleen Mullen, and Alexander Strand, "Does Delay Cause Decay? The Effect of Administrative Decision Time on the Labor Force Participation and Earnings of Disability Applicants," 2015.

Bertrand, Marianne, Erzo F.P. Luttmer, and Sendhil Mullainathan, "Network Effects and Welfare Cultures," Quarterly Journal of Economics, 2000, 115 (3), 1019-1055.

_, Sendhil Mullainathan, and Eldar Shafir, "A Behavioral-Economics View of Poverty," American Economic Review: Papers 63 Proceedings, 2004, 94 (2), 419-423.

Besley, Timothy and Stephen Coate, "Workfare versus Welfare: Incentive Arguments for Work Requirements in Poverty-Alleviation Programs," American Economic Review, 1992, 82 (1), 249261.

Bettinger, E. P., B. Terry Long, P. Oreopoulos, and L. Sanbonmatsu, "The Role of Application Assistance and Information in College Decisions: Results from the H\&R Block FAFSA Experiment," The Quarterly Journal of Economics, 2012, pp. 1205-1242.

Black, Dan, Kermit Daniel, and Seth Sanders, "The Impact of Economic Conditions on Participation in Disability Programs: Evidence from the Coal Boom and Bust," American Economic Review, 2002, 92 (1), 27-50.

Chetty, Raj, John N. Friedman, and Emmanuel Saez, "Using differences in knowledge across neighborhoods to uncover the impacts of the EITC on earnings," American Economic Review, 2013, 103 (7), 2683-2721.

Cohen, Jessica, Pascaline Dupas, and Simone Schaner, "Price Subsidies, Diagnostic Tests, and Targeting of Malaria Treatment: Evidence from a Randomized Controlled Trial," American Economic Review, 2015, 105 (2), 609-645. 
Currie, Janet, "The Take Up of Social Benefits," 2004.

Dahl, Gordon B, Andreas Kostol, and Magne Mogstad, "Family Welfare Cultures," Quarterly Journal of Economics, 2014, 129 (4), 1711-1752.

Duggan, Mark and Scott A Imberman, "Why Are the Disability Rolls Skyrocketing? The Contribution of Population Characteristics, Economic Conditions, and Program Generosity," in "Health at Older Ages: The Causes and Consequences of Declining Disability among the Elderly" number January 2009, pp. 337-379.

Ebenstein, Avraham and Kevin Stange, "Does Inconvenience Explain Low Take-Up? Evidence from Unemployment Insurance," Journal of Policy Analysis and Management, 2010, 29 (1), 111136.

Einav, Liran, Amy Finkelstein, and Mark R. Cullen, "Estimating Welfare in Insurance Markets Using Variation in Prices," Quarterly Journal of Economics, 2010, 125 (3), 877-921.

Fadlon, Itzik and Torben Heien Nielsen, "Household Responses to Severe Health Shocks and the Design of Social Insurance," 2015.

Finkelstein, Amy, Nathaniel Hendren, and Erzo F.P. Luttmer, "The Value of Medicaid: Welfare Analysis of Public Health Insurance Expansions," 2015.

Golosov, Mikhail and Aleh Tsyvinski, "Designing Optimal Disability Insurance: A Case for Asset Testing," Journal of Political Economy, 2006, 114 (2), 257-279.

Gruber, Jonathan, Phillip Levine, and Douglas Staiger, "Abortion Legalization and Child Living Circumstances: Who is the âMarginal Childâ?," The Quarterly Journal of Economics, 1999, 114 (1), 263-291.

Guryan, Jonathan, "Desegregation and Black Dropout Rates," American Economic Review, 2004, $94(4), 919-943$.

Kleven, Henrik Jacobsen and Wojciech Kopczuk, "Transfer Program Complexity and the Take Up of Social Benefits," American Economic Journal: Economic Policy, 2011, 3 (1), 54-90.

Kopczuk, Wojciech and Cristian Pop-Eleches, "Electronic Filing, Tax Preparers and Participation in the Earned Income Tax Credit," Journal of Public Economics, 2007, 91 (7-8), 1351-1367. 
Madrian, Brigitte C. and Dennis F. Shea, "The Power of Suggestion: Inertia in 401(k) Participation and Savings Behavior," Quarterly Journal of Economics, 2001, CXVI (4), 1149-1187.

Maestas, Nicole, Kathleen J Mullen, and Alexander Strand, "Does Disability Insurance Receipt Discourage Work? Using Examiner Assignment to Estimate Causal Effects," American Economic Review, 2013, 103 (5), 1797-1829.

Meyer, Bruce D. and Wallace K.C. Mok, "Disability, Earnings, Income, and Consumption," 2013.

Nichols, Albert L. and Richard J. Zeckhauser, "Targeting Transfers through Restrictions on Recipients," American Economic Review, 1982, 72 (2), 372-377.

Nichols, D., E Smolensky, and T N Tideman, "Discrimination by Waiting Time in Merit Goods," American Economic Review, 1971, 61 (3), 312-323.

Rossin-Slater, Maya, "WIC in Your Neighborhood: New Evidence on the Impacts of Geographic Access to Clinics," Journal of Public Economics, 2013, 102 (March), 51-69. 


\section{Main Figures and Tables}

Figure 1: Timing of Field Office Closings

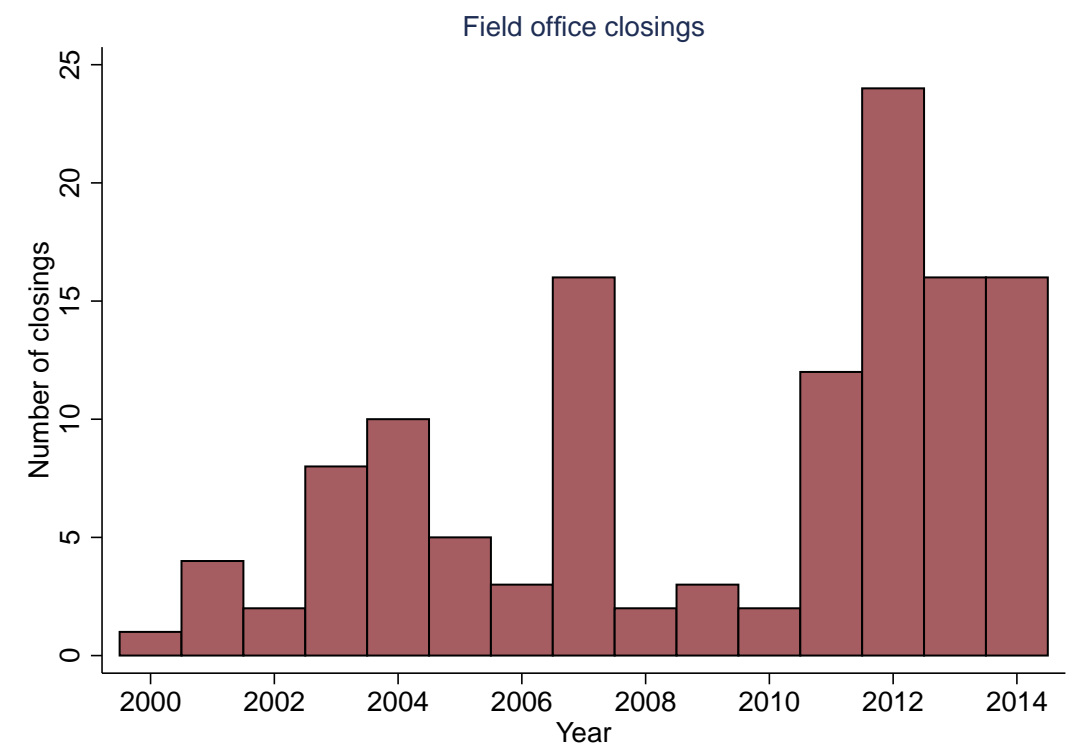

Source: Authors' tabulations based on Social Security Administration data. 
Figure 2: Map of Field Office Closings and ZIP Classification in United States

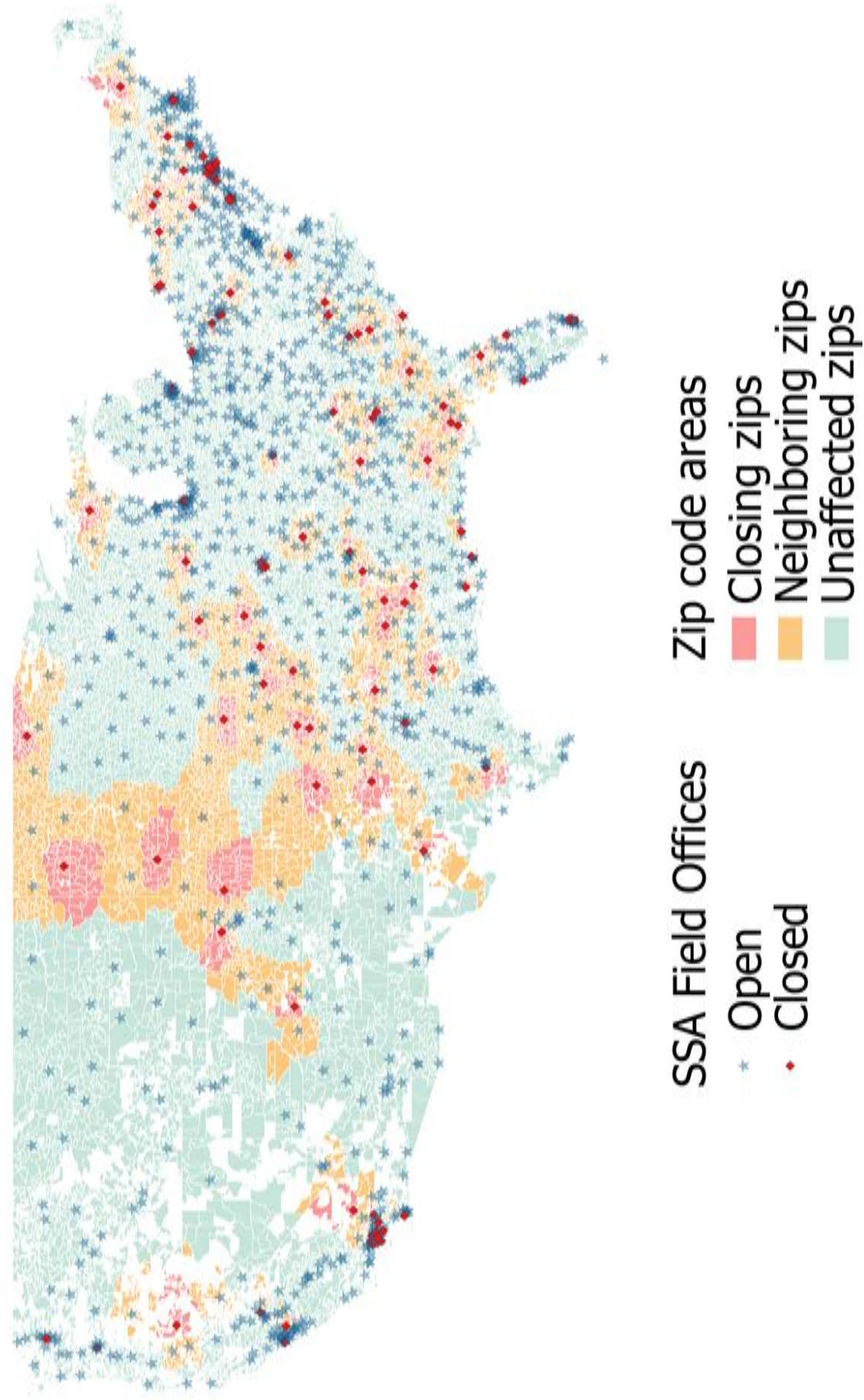


Figure 3: Raw Plots of Number of Applications in Control and Treatment ZIPs

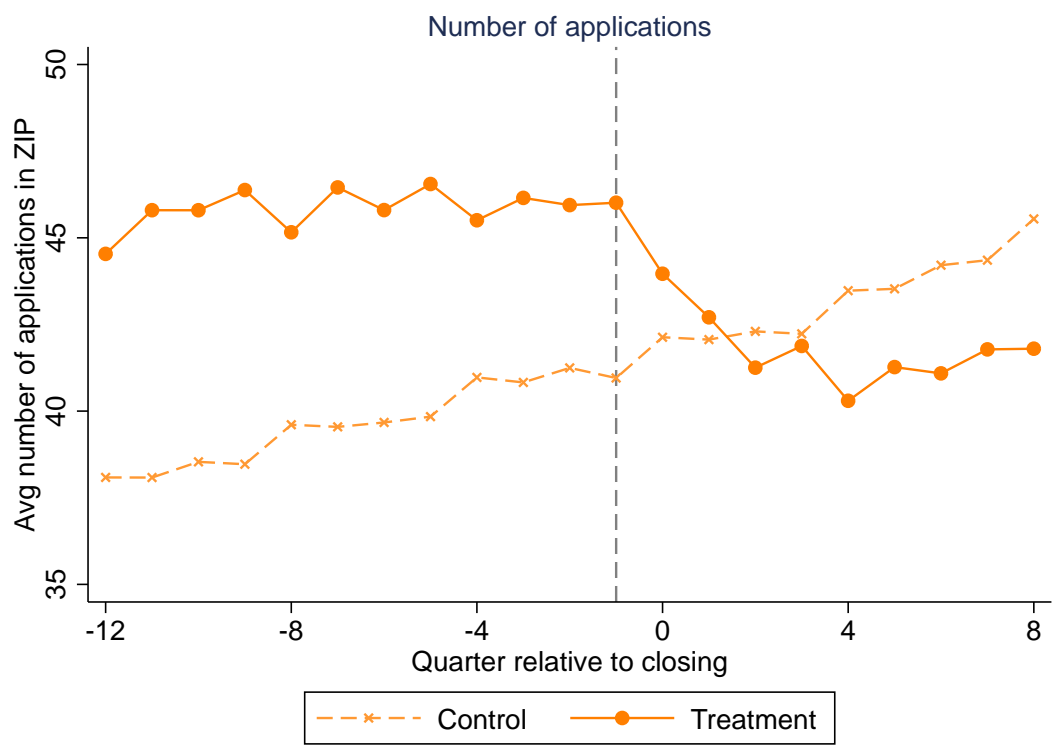

Notes: Figure plots raw (non-regression-adjusted) counts of applications in control and treatment ZIPs relative to the quarter of the closing. Sample is ZIP codes whose nearest office closes after 2000 and that have an average of at least three disability applications per quarter in the year before the closing. Treatment ZIPs are ZIPs whose nearest office closes for a given closing, while control ZIPs are ZIPs whose nearest office closes in a future closing.

Figure 4: Effect of Closings on Number of Disability Applications and Allowances

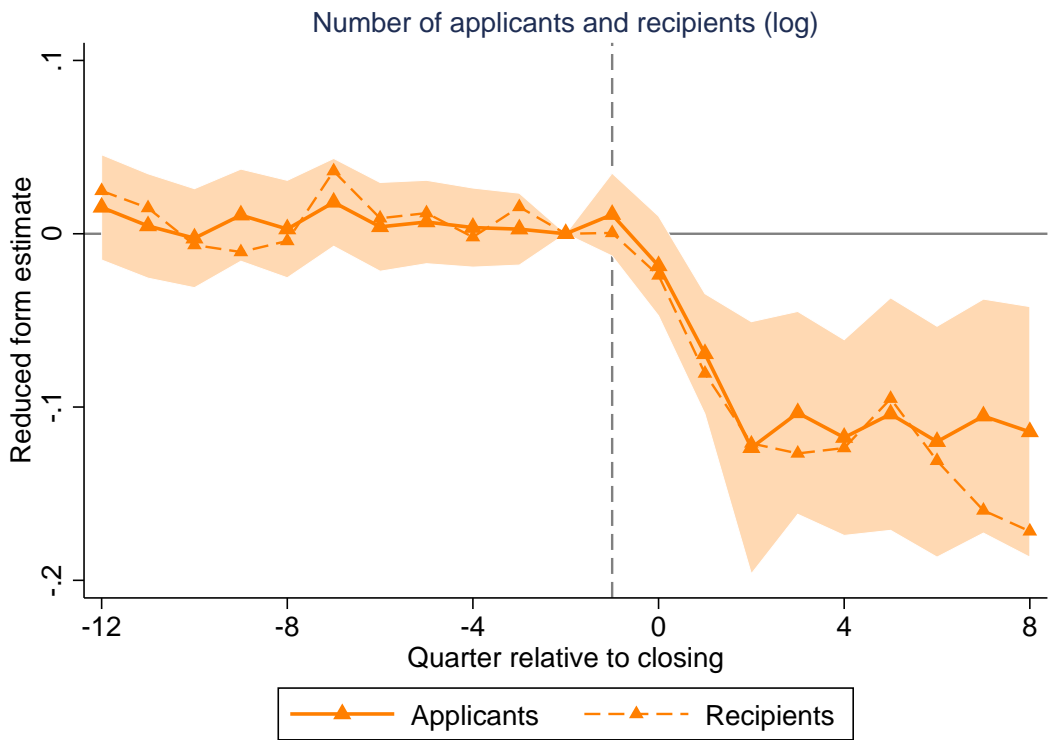

Notes: Figure plots estimates of $\delta_{\tau}$ coefficients from equation (1), where the dependent variable is the log number of disability applications (solid series) or the log number of disability recipients (dashed series). Shaded region is 95 percent confidence interval for disability applications (solid series). Sample is ZIP codes whose nearest office closes after 2000 and that have an average of at least three disability applications per quarter in the year before the closing. Regressions are weighted by application or recipient volume in the year before the closing. 
Figure 5: Effect of Closings on Number of Disability Applications, by Subgroup
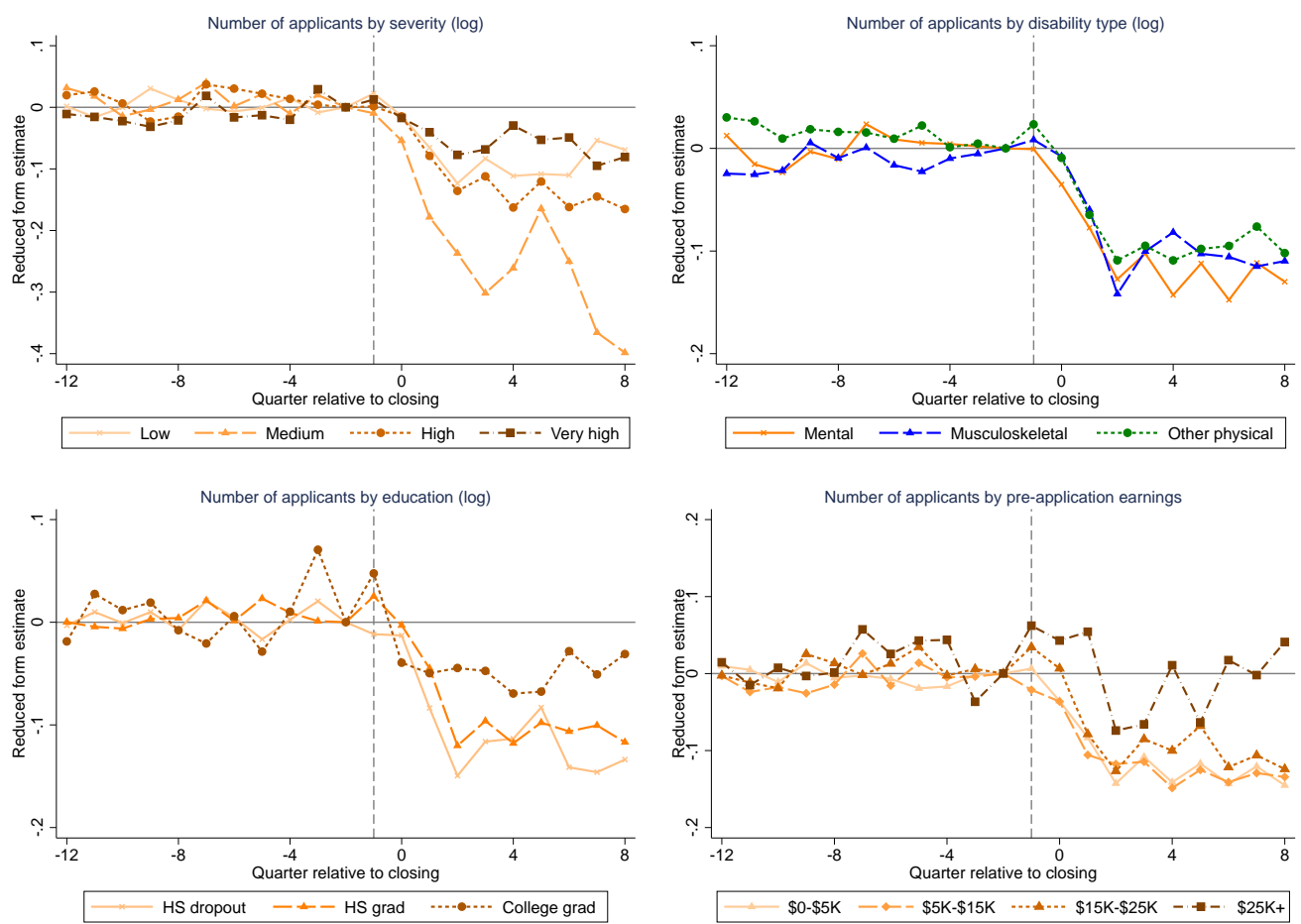

Notes: Figure plots estimates of $\delta_{\tau}$ coefficients from equation (1), where the dependent variable is the log number of disability applications by subgroup. Sample is ZIP codes whose nearest office closes after 2000 and that have an average of at least three disability applications per quarter in the year before the closing. Regressions are weighted by application volume in the year before the closing.

Figure 6: Robustness: Event Study Specifications, with and without Unaffected ZIPs
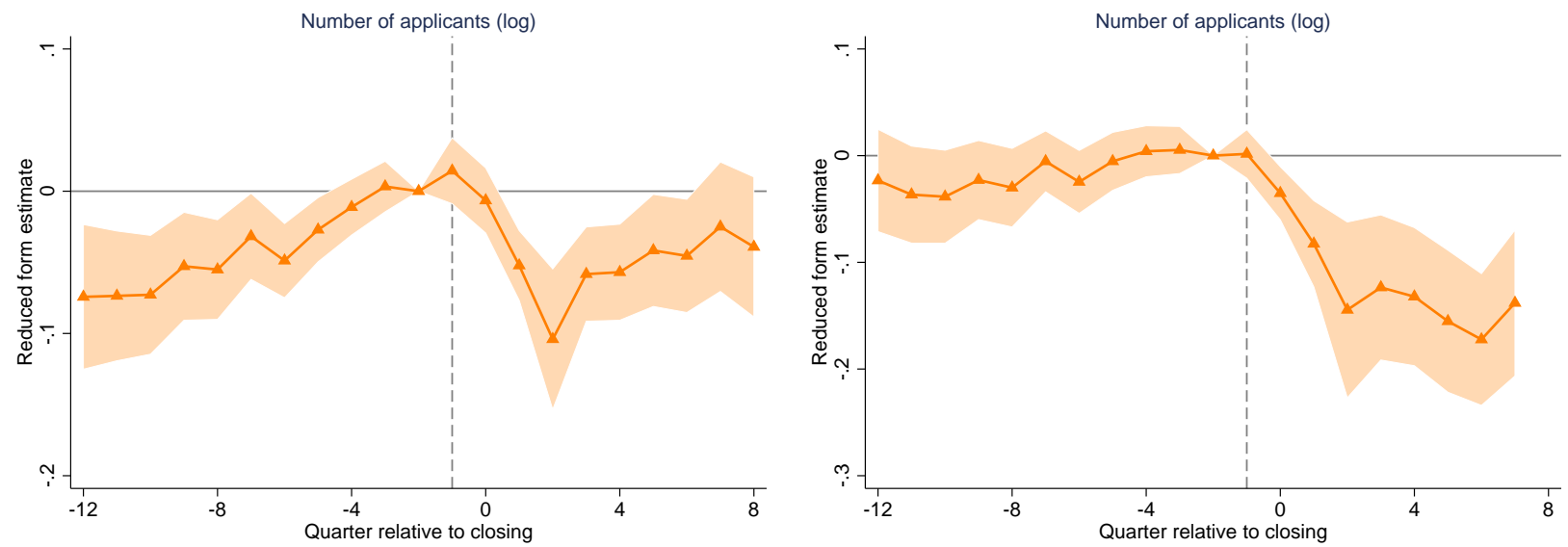

Notes: Figures plot estimates of $\delta_{\tau}$ coefficients from equation (5), where the dependent variable is the log number of disability applications. The left graph includes unaffected ZIPs as controls, while the right graph includes only closing ZIPs. For both, the sample contains only ZIPs with an average of at least three disability applications per quarter in the year before the closing. Regressions are weighted by application volume in the year before the closing. 
Figure 7: Model of Non-Monotonic Effects by Severity
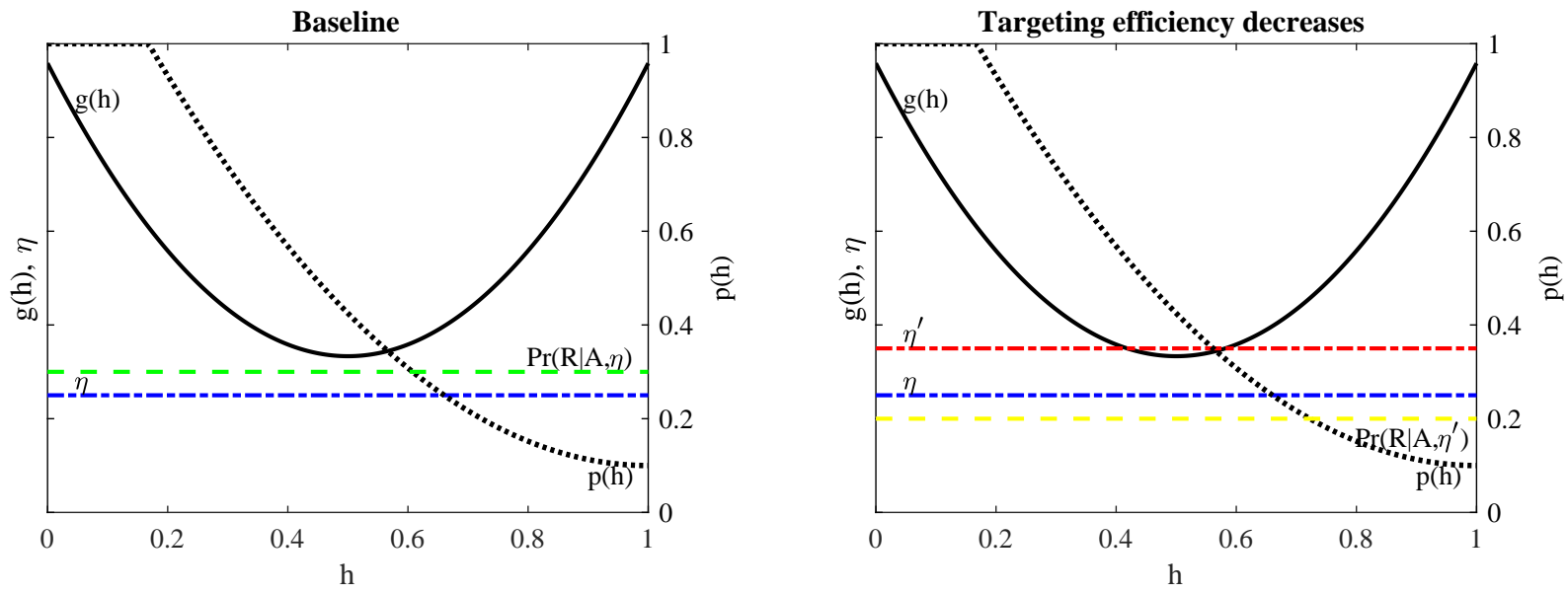

Notes: Figure presents a model that explains non-monotonic effects by severity. The left-hand graph shows baseline conditions, with health $h$ on the x-axis, where $h=1$ is the best health. Measured on the right axis are the individual probability of allowance $p(h)$ and the average probability of allowance for those who apply $\operatorname{Pr}(R \mid A, \eta)$. Measured on the left axis are the benefits of application $g(h)$ and the line $\eta$ is the cost of application. As explained in Section 5.B, the function $g(h)$ is non-monotonic because poor-health (highseverity) applicants value benefits because of poor health, while good-health (low-severity) applicants value benefits because they are negatively selected on skills. At baseline, everyone applies since $g(h)$ is everywhere above $\eta$. The right-hand graph shows what happens when application costs increase from $\eta$ to $\eta^{\prime}$. Individuals in the middle of the health distribution are screened out, while those at either end continue applying.

Figure 8: Effect of Closings on Measures of Field Office Congestion
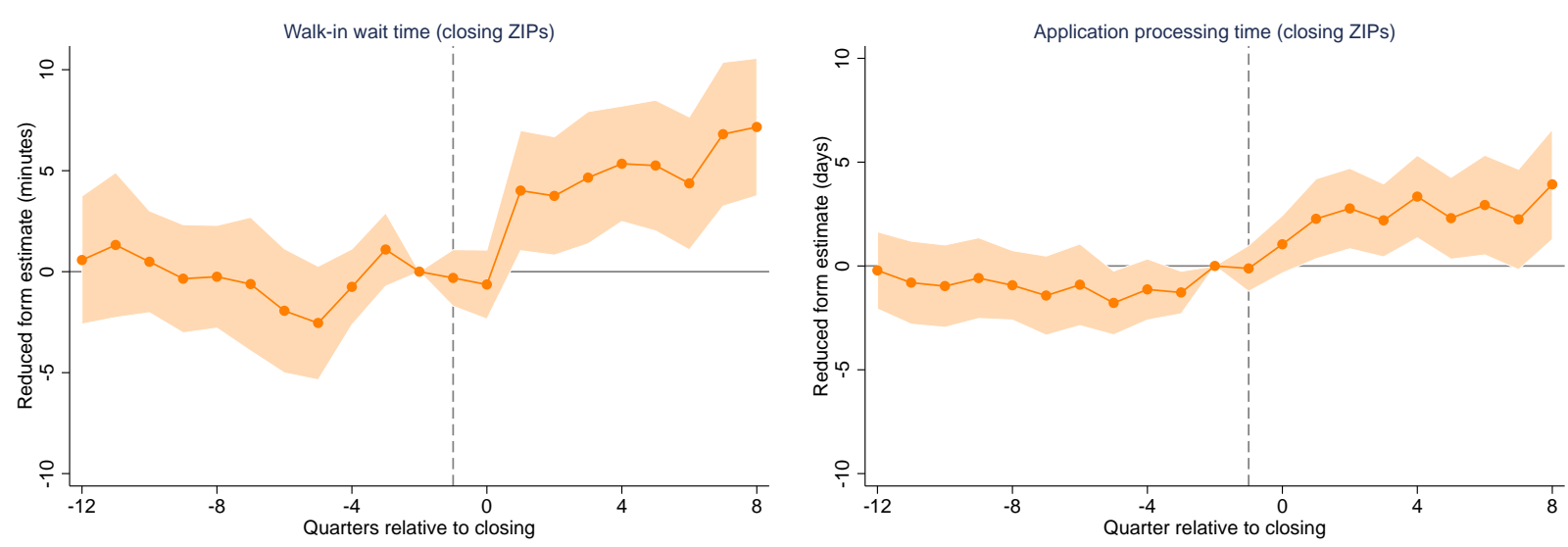

Notes: Figure plots estimates of $\beta_{\tau}$ coefficients from equation (1), where the dependent variable is average walk-in wait time in minutes at nearest field office (left) or the average number of days it takes the field office to process a disability application (right). Shaded region is 95 percent confidence interval. Sample is ZIP codes whose nearest office closes after 2000 and that have an average of at least three disability applications per quarter in the year before the closing. Regressions are weighted by application volume in the year before the closing. 
Table 1: Summary Statistics of Closing, Neighboring, and Unaffected ZIP Codes in Sample

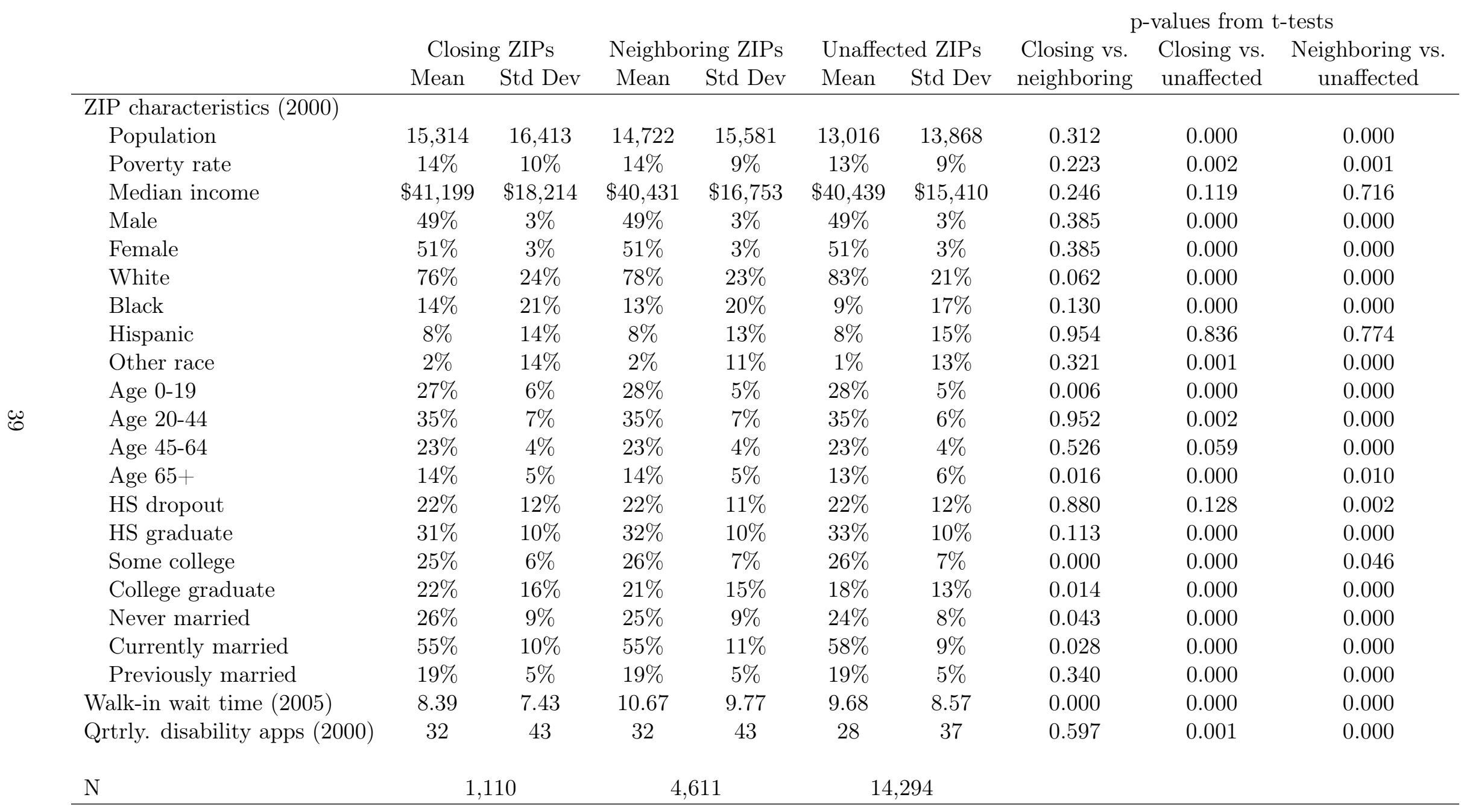

Notes: Table presents summary statistics for ZIP codes with an average of at least three disability applications per quarter in the year 2000. Closing ZIPs are ZIPs whose closest office closes. Neighboring ZIPs are ZIPs whose closest office is the second or third closest office to a closing ZIP. Unaffected ZIPs are ZIPs that are neither closing nor neighboring ZIPs. "ZIP characteristics" are calculated from the 2000 Census, "walk-in wait time" from Social Security Administration data (where 2005 is the earliest available year), and "quarterly disability applications" from Social Security Administration data. 
Table 2: Estimates of the Effect of Closings on Disability Applications

\begin{tabular}{|c|c|c|c|c|c|c|}
\hline & \multicolumn{3}{|c|}{ Count (log) } & \multicolumn{3}{|c|}{ Proportion/average } \\
\hline & $\begin{array}{l}\text { Pt. } \\
\text { Est. }\end{array}$ & $\begin{array}{l}\text { Std. } \\
\text { Err. }\end{array}$ & $\begin{array}{c}\text { Cntrl. } \\
\text { Ct. }\end{array}$ & $\begin{array}{l}\text { Pt. } \\
\text { Est. }\end{array}$ & $\begin{array}{l}\text { Std. } \\
\text { Err. }\end{array}$ & $\begin{array}{l}\text { Cntrl. } \\
\text { Mean }\end{array}$ \\
\hline All & $-0.110^{* * *}$ & $(0.0300)$ & 39.7 & & & \\
\hline \multicolumn{7}{|l|}{ Severity } \\
\hline Low & $-0.0841^{* *}$ & $(0.0322)$ & 18.0 & $0.0146^{* *}$ & $(0.00601)$ & 0.425 \\
\hline Medium & $-0.269^{* * *}$ & $(0.0518)$ & 6.9 & $-0.0146^{* * *}$ & $(0.00349)$ & 0.184 \\
\hline High & $-0.160 * * *$ & $(0.0393)$ & 8.5 & $-0.00879^{* *}$ & $(0.00419)$ & 0.209 \\
\hline Very high & -0.0473 & $(0.0302)$ & 6.2 & $0.00877^{* * *}$ & $(0.00243)$ & 0.183 \\
\hline \multicolumn{7}{|l|}{ Disability type } \\
\hline Mental & $-0.116^{* * *}$ & $(0.0351)$ & 12.3 & -0.00334 & $(0.00434)$ & 0.289 \\
\hline Musculoskeletal & $-0.0654^{* *}$ & $(0.0318)$ & 10.2 & $0.00829^{* * *}$ & $(0.00272)$ & 0.276 \\
\hline Other physical & $-0.116^{* * *}$ & $(0.0309)$ & 17.2 & -0.00495 & $(0.00452)$ & 0.435 \\
\hline Education (years) & & & & $0.0591^{* *}$ & $(0.0265)$ & 11.8 \\
\hline HS dropout & $-0.129^{* * *}$ & $(0.0315)$ & 9.9 & & & \\
\hline HS graduate & $-0.0879^{* * *}$ & $(0.0264)$ & 19.4 & & & \\
\hline College graduate & -0.0397 & $(0.0311)$ & 2.4 & & & \\
\hline Pre-application earnings $(\$)$ & & & & $654.1^{* * *}$ & $(216.2)$ & $\$ 15,362$ \\
\hline$\$ 0-\$ 5,000$ & $-0.130^{* * *}$ & $(0.0332)$ & 18.7 & & & \\
\hline$\$ 5,000-\$ 15,000$ & $-0.101 * * *$ & $(0.0319)$ & 8.9 & & & \\
\hline$\$ 15,000-\$ 25,000$ & $-0.0893^{* * *}$ & $(0.0307)$ & 5.0 & & & \\
\hline$\$ 25,000+$ & -0.0112 & $(0.0396)$ & 7.0 & & & \\
\hline \multicolumn{7}{|l|}{ Language } \\
\hline Speaks English & -0.0788 & $(0.0623)$ & 24.9 & $-0.0158^{* * *}$ & $(0.00575)$ & 0.623 \\
\hline Does not speak English & 0.00240 & $(0.0663)$ & 14.7 & & & \\
\hline Age (years) & & & & 0.232 & $(0.149)$ & 40.7 \\
\hline $18-34$ & $-0.117^{* * *}$ & $(0.0350)$ & 7.9 & & & \\
\hline $35-49$ & $-0.116^{* * *}$ & $(0.0318)$ & 12.9 & & & \\
\hline $50+$ & $-0.0882^{* * *}$ & $(0.0266)$ & 13.1 & & & \\
\hline \multicolumn{7}{|l|}{ Applicant behavior } \\
\hline Files online & 0.0708 & -0.0565 & 2.8 & $0.0268^{* * *}$ & $(0.00859)$ & 0.075 \\
\hline Files in person or by phone & $-0.150^{* * *}$ & -0.0337 & 36.9 & & & \\
\hline Provides email address & 0.0744 & $(0.0608)$ & 4.2 & $0.0190^{*}$ & $(0.00983)$ & 0.111 \\
\hline No email address & $-0.130 * * *$ & $(0.0341)$ & 35.4 & & & \\
\hline Has representation & 0.0514 & $(0.0692)$ & 2.2 & 0.00450 & $(0.00525)$ & 0.054 \\
\hline No representation & $-0.115^{* * *}$ & $(0.0313)$ & 37.4 & & & \\
\hline
\end{tabular}

Notes: ${ }^{* *} \mathrm{p}<0.01,{ }^{* *} \mathrm{p}<0.05,{ }^{*} \mathrm{p}<0.1$. The first set of columns presents estimates of the effect of field office closings on $\log$ applications by subgroup, specifically estimates of $\beta$ from equation (2). The second set of columns presents estimates of $\beta$ where the dependent variable is the proportion of applicants with that characteristic (for indicator variables like severity, disability type, applicant behavior, and language) or the average of the characteristic across applicants (for continuous variables like education, earnings, and age). If some subgroups are small, the change in proportion may be small even when there is substantial heterogeneity in the effects across subgroups. Earnings and education estimates include only adult applicants. The "Control Count" is the number of individuals in the control ZIP in a category, and "Control Mean" is the mean characteristic in the control group. Sample is ZIP codes whose nearest office closes after 2000 and that have an average of at least three disability applications per quarter in the year before the closing. Regressions are weighted by application volume in the year before the closing. Standard errors in parentheses. 
Table 3: Estimates of the Effect of Closings on Disability Receipt

\begin{tabular}{|c|c|c|c|c|c|c|}
\hline & \multicolumn{3}{|c|}{ Count (log) } & \multicolumn{3}{|c|}{ Proportion/average } \\
\hline & Pt. & Std. & Cntrl. & Pt. & Std. & Cntrl. \\
\hline All & $-0.133^{* * *}$ & $(0.0312)$ & 21.7 & & & \\
\hline Severity & & & & & & \\
\hline Low & & $\mathrm{N} / \mathrm{A}$ & & & $\mathrm{N} / \mathrm{A}$ & \\
\hline Medium & $-0.244^{* * *}$ & $(0.0478)$ & 6.9 & $-0.0257^{* * *}$ & $(0.00570)$ & 0.329 \\
\hline High & $-0.148^{* * *}$ & $(0.0371)$ & 8.5 & -0.00647 & $(0.00538)$ & 0.359 \\
\hline Very high & -0.0407 & $(0.0286)$ & 6.2 & $0.0322^{* * *}$ & $(0.00601)$ & 0.312 \\
\hline Disability type & & & & & & \\
\hline Mental & $-0.151^{* * *}$ & $(0.0341)$ & 6.9 & $-0.00897^{* *}$ & $(0.00393)$ & 0.289 \\
\hline Musculoskeletal & $-0.108^{* * *}$ & $(0.0358)$ & 5.1 & 0.00120 & $(0.00352)$ & 0.252 \\
\hline Other physical & $-0.110^{* * *}$ & $(0.0311)$ & 9.7 & $0.00777^{*}$ & $(0.00457)$ & 0.459 \\
\hline Education (years) & & & & 0.0265 & $(0.0316)$ & 11.9 \\
\hline HS dropout & $-0.123^{* * *}$ & $(0.0338)$ & 5.1 & & & \\
\hline HS graduate & $-0.123^{* * *}$ & $(0.0298)$ & 10.6 & & & \\
\hline College graduate & -0.0507 & $(0.0317)$ & 1.6 & & & \\
\hline Pre-application earnings $(\$)$ & & & & $821.1^{* * *}$ & $(271.7)$ & $\$ 18,328$ \\
\hline$\$ 0-\$ 5,000$ & $-0.140^{* * *}$ & $(0.0340)$ & 9.0 & & & \\
\hline$\$ 5,000-\$ 15,000$ & $-0.142^{* * *}$ & $(0.0368)$ & 4.5 & & & \\
\hline$\$ 15,000-\$ 25,000$ & $-0.116^{* * *}$ & $(0.0332)$ & 3.1 & & & \\
\hline$\$ 25,000+$ & -0.0451 & $(0.0342)$ & 5.1 & & & \\
\hline Age (years) & & & & 0.236 & $(0.177)$ & 43.0 \\
\hline $18-34$ & $-0.133^{* * *}$ & $(0.0357)$ & 3.1 & & & \\
\hline $35-49$ & $-0.185^{* * *}$ & $(0.0396)$ & 6.1 & & & \\
\hline $50+$ & $-0.107 * * *$ & $(0.0290)$ & 9.3 & & & \\
\hline
\end{tabular}

Notes: $* * * \mathrm{p}<0.01,{ }^{* *} \mathrm{p}<0.05, * \mathrm{p}<0.1$. The first set of columns presents estimates of the effect of field office closings on log allowances by subgroup, specifically estimates of $\beta$ from equation (2). The second set of columns presents estimates of $\beta$ where the dependent variable is the proportion of recipients with that characteristic (for indicator variables like severity, disability type, applicant behavior, and language) or the average of the characteristic across recipients (for continuous variables like education, earnings, and age). Earnings and education estimates include only adult allowances. The "Control Count" is the number of individuals in the control ZIP in a category, and "Control Mean" is the mean characteristic in the control group. "Low" severity not applicable at the allowance level because low severity is defined as being denied. Sample is ZIP codes whose nearest office closes after 2000 and that have an average of at least three disability applications per quarter in the year before the closing. Regressions are weighted by recipient volume in the year before the closing. Standard errors in parentheses. 
Table 4: Estimates of the Effect of Closings on Types of Application Costs

\begin{tabular}{lcccccc} 
& \multicolumn{3}{c}{ Closing ZIP } & \multicolumn{3}{c}{ Neighboring ZIP } \\
& Pt. Est. & Std. Err. & Mean & Pt. Est. & Std. Err. & Mean \\
\hline Applications (log) & $-0.110^{* * *}$ & $(0.0300)$ & 39.7 & $-0.0539^{* * *}$ & $(0.0176)$ & 42.5 \\
Recipients (log) & $-0.133^{* * *}$ & $(0.0312)$ & 21.7 & $-0.0904^{* * *}$ & $(0.0181)$ & 22.6 \\
Congestion measures & & & & & & \\
$\quad$ FO processing time & $3.032^{* * *}$ & $(1.094)$ & 28.8 & $2.804^{* * *}$ & $(0.731)$ & 28.4 \\
$\quad$ Walk-in wait times & $4.352^{* * *}$ & $(1.412)$ & 13.6 & $3.472^{* * *}$ & $(1.126)$ & 16.3 \\
Travel cost measures & & & & & & \\
$\quad$ Driving time & $9.974^{* * *}$ & $(1.636)$ & 23.5 & & & \\
$\quad$ Driving distance & $11.98^{* * *}$ & $(1.338)$ & 24.3 & & & \\
$\quad$ Transit time & $35.76^{* * *}$ & $(6.426)$ & 89.4 & & & \\
\hline
\end{tabular}

Notes: ${ }^{* * *} \mathrm{p}<0.01,{ }^{* *} \mathrm{p}<0.05,{ }^{*} \mathrm{p}<0.1$. Table presents estimates of the effect of field office closings on log applications, $\log$ allowances, and measures of application costs for closing and neighboring ZIPs, specifically estimates of $\beta$ from equation (2), where Treated $_{i c}$ is replaced with TreatedNbric for the neighboring ZIP regressions. A closing ZIP is a ZIP whose nearest office closes. A neighboring ZIP is a ZIP whose nearest office is the second or third closest office of a closing ZIP. Walk-in wait time is the average time (in minutes) that a visitor to a field office waits to be seen. Processing time is the number of days it takes a field office to send an application to a state disability determination services office. Driving time, driving distance, and public transit time to the nearest field office are calculated using Google maps with the trip originating from the ZIP centriod. Sample is ZIP codes whose nearest office closes after 2000 and that have an average of at least three disability applications per quarter in the year before the closing. Regressions are weighted by application or recipient volume in the year before the closing. Standard errors in parentheses. 
Table 5: IV Estimates of the Effect of Different Application Costs on Disability Applications

\begin{tabular}{|c|c|c|c|c|c|c|c|c|}
\hline & \multicolumn{2}{|c|}{ First Stage } & \multirow{2}{*}{$\begin{array}{l}\text { Red. Form } \\
\text { Log(app) }\end{array}$} & \multirow{2}{*}{$\begin{array}{c}\text { OLS } \\
\log (\text { app })\end{array}$} & \multirow{2}{*}{$\begin{array}{c}\text { IV } \\
\log (\text { app })\end{array}$} & \multirow{2}{*}{$\begin{array}{l}\text { Inc after } \\
\text { closing }\end{array}$} & \multirow{2}{*}{$\begin{array}{c}\text { OLS } \Delta \text { in } \\
\log (\text { app })\end{array}$} & \multirow{2}{*}{$\begin{array}{l}\text { IV } \Delta \text { in } \\
\log (\text { app })\end{array}$} \\
\hline & Driving dist & Wait time & & & & & & \\
\hline TrtXPostXDDiff & $\begin{array}{l}0.996^{* * *} \\
(0.00442)\end{array}$ & & $\begin{array}{l}-0.00320 \\
(0.00250)\end{array}$ & & & & & \\
\hline TrtXPostXWDiff & & $\begin{array}{c}0.452^{* * *} \\
(0.0981)\end{array}$ & $\begin{array}{l}-0.000454 \\
(0.000588)\end{array}$ & & & & & \\
\hline NbrXPost & & $\begin{array}{c}4.564^{* * *} \\
(1.168)\end{array}$ & $\begin{array}{c}-0.0781^{* * *} \\
(0.0107)\end{array}$ & & & & & \\
\hline New Office (TrtXPost) & & & $\begin{array}{c}-0.148^{* * *} \\
(0.0348)\end{array}$ & $\begin{array}{c}-0.0830^{* * *} \\
(0.0246)\end{array}$ & $\begin{array}{l}-0.0229 \\
(0.0221)\end{array}$ & 1 & -0.083 & -0.023 \\
\hline Driving distance $(\mathrm{km})$ & & & & $\begin{array}{l}-0.000483 \\
(0.000431)\end{array}$ & $\begin{array}{l}-0.000253 \\
(0.000408)\end{array}$ & 12 & -0.006 & -0.003 \\
\hline Wait time (min) & & & & $\begin{array}{c}-0.00273^{* * *} \\
(0.000647)\end{array}$ & $\begin{array}{c}-0.0158^{* * *} \\
(0.00327)\end{array}$ & 4.3 & -0.012 & -0.068 \\
\hline $\mathrm{N}$ & 101,008 & 80,779 & 98,557 & 77,786 & 76,280 & & & \\
\hline
\end{tabular}

Notes: ${ }^{* * *} \mathrm{p}<0.01,{ }^{* *} \mathrm{p}<0.05,{ }^{*} \mathrm{p}<0.1$. Table presents first stage estimates from equations (8) and (9), reduced form estimates, and OLS and IV estimates from equation (7). The first stage for wait time gives the effect of the difference in pre-closing wait time of the now-closest office and previously-closest office on wait time (in minutes), and analogously for the first stage for distance (in kilometers). The reduced form estimates give the effect of the instruments on $\log$ disability applications. The IV estimates give the effect of wait time, distance, and office switching on log disability applications. Sample is ZIP codes whose nearest office closes after 2000 and that have an average of at least three disability applications per quarter in the year before the closing. Wait time data are not available for all offices in all quarters, so the sample size falls when wait time is included in the regression. Regressions are weighted by application volume in the year before the closing. Standard errors in parentheses. 
Table 6: Costs and Benefits of Field Office Closings

$\begin{array}{lr}\text { Costs of closing (thousands) } & \\ \text { Lower receipt in areas surrounding closed office } & \$ 3,100 \\ \text { Lower receipt in areas surrounding neighboring office } & \$ 13,000 \\ \text { Higher applicant time and earnings decay } & \$ 3,200 \\ \text { Total } & \$ 19,300 \\ & \\ \text { Benefits of closing (thousands) } & \$ 2,600 \\ \text { Administrative savings from processing fewer applications } & \$ 500 \\ \text { Administrative savings from closing field office } & \$ 1,000 \\ \text { Application cost savings from discouraged applicants } & \$ 4,100 \\ \text { Total } & 5 \\ \text { Ratio of costs to benefits } & \end{array}$

Notes: Table presents estimates of costs and benefits of field office closings, in thousands of dollars. See Appendix for detailed explanation of calculations. 